\title{
HELIOS: AN OPEN-SOURCE, GPU-ACCELERATED RADIATIVE TRANSFER CODE FOR SELF-CONSISTENT EXOPLANETARY ATMOSPHERES
}

\author{
Matej Malik ${ }^{1}$, Luc Grosheintz ${ }^{1}$, João M. Mendonça ${ }^{1}$, Simon L. Grimm ${ }^{1,2}$, Baptiste Lavie ${ }^{1}$, \\ Daniel Kitzmann ${ }^{1}$, Shang-Min Tsai ${ }^{1}$, Adam Burrows ${ }^{3}$, Laura Kreidberg ${ }^{4,5}$, Megan Bedell ${ }^{4}$, \\ Jacob L. Bean ${ }^{4}$, Kevin B. Stevenson ${ }^{4}$, and Kevin Heng ${ }^{1}$ \\ ${ }^{1}$ University of Bern, Center for Space and Habitability, Sidlerstrasse 5, CH-3012, Bern, Switzerland; \\ matej.malik@csh.unibe.ch, kevin.heng@csh.unibe.ch \\ ${ }^{2}$ University of Zürich, Institute for Computational Science, Winterthurerstrasse 190, CH-8057 Zürich, Switzerland \\ ${ }^{3}$ Department of Astrophysical Sciences, Princeton University Peyton Hall, Princeton, NJ 08544, USA \\ ${ }^{4}$ Department of Astronomy and Astrophysics, University of Chicago, 5640 S. Ellis Avenue, Chicago, IL 60637, USA \\ ${ }^{5}$ Harvard-Smithsonian Center for Astrophysics, 60 Garden Street, Cambridge, MA 02138, USA \\ Received 2016 May 26; revised 2016 November 19; accepted 2016 November 30; published 2017 January 9
}

\begin{abstract}
We present the open-source radiative transfer code named HELIOS, which is constructed for studying exoplanetary atmospheres. In its initial version, the model atmospheres of HELIOS are one-dimensional and planeparallel, and the equation of radiative transfer is solved in the two-stream approximation with nonisotropic scattering. A small set of the main infrared absorbers is employed, computed with the opacity calculator HELIOS$\mathrm{K}$ and combined using a correlated- $k$ approximation. The molecular abundances originate from validated analytical formulae for equilibrium chemistry. We compare HELIOS with the work of Miller-Ricci \& Fortney using a model of GJ 1214b, and perform several tests, where we find: model atmospheres with single-temperature layers struggle to converge to radiative equilibrium; $k$-distribution tables constructed with $\gtrsim 0.01 \mathrm{~cm}^{-1}$ resolution in the opacity function $\left(\lesssim 10^{3}\right.$ points per wavenumber bin) may result in errors $\gtrsim 1 \%-10 \%$ in the synthetic spectra; and a diffusivity factor of 2 approximates well the exact radiative transfer solution in the limit of pure absorption. We construct "null-hypothesis" models (chemical equilibrium, radiative equilibrium, and solar elemental abundances) for six hot Jupiters. We find that the dayside emission spectra of HD 189733b and WASP-43b are consistent with the null hypothesis, while the latter consistently underpredicts the observed fluxes of WASP-8b, WASP-12b, WASP-14b, and WASP-33b. We demonstrate that our results are somewhat insensitive to the choice of stellar models (blackbody, Kurucz, or PHOENIX) and metallicity, but are strongly affected by higher carbon-to-oxygen ratios. The code is publicly available as part of the Exoclimes Simulation Platform (exoclime.net).
\end{abstract}

Key words: methods: numerical - planets and satellites: atmospheres - radiative transfer

\section{INTRODUCTION}

The past few years have been marked by a slow, but steady, shift from the era of the detections of exoplanets to the new age of the characterization of their atmospheres. Exoplanets transiting in front of their host stars allow for atmospheric features to be imprinted onto the total system light (Seager \& Sasselov 2000; Brown et al. 2001; Charbonneau et al. 2002). Secondary eclipses allow for photons from the exoplanetary atmosphere to be directly measured (Charbonneau et al. 2005; Deming et al. 2005). Extracting the spectroscopic signatures of these exoplanetary atmospheres is a challenging task, because they are typically many orders of magnitude fainter than the light from their host stars. Interpreting these signatures requires a profound understanding of radiative transfer and atmospheric chemistry, in order to infer the thermal structure and atomic/molecular abundances of the atmosphere from the data.

Hot Jupiters are particularly accessible to atmospheric characterization via transits and eclipses. They are hardly 1D objects, but a reasonable first approach is to study them using 1D, plane-parallel model atmospheres (Sudarsky et al. 2003; Barman et al. 2005; Fortney et al. 2005, 2006, 2008, 2010; Burrows et al. 2006, 2007, 2008), which may be used to mimic the dayside- or nightside-integrated emission. The simplest model one may construct of a dayside emission spectrum (besides a Planck function) is a 1D model with an atmosphere in radiative and chemical equilibrium, if one neglects the effects of atmospheric dynamics and photochemistry. Despite these simplifications, there are several nontrivial demands associated with such a model: it should be able to consider a rich variety of chemistries, metallicities, irradiation fluxes from the star, and internal heat fluxes from the interior of the exoplanet. It should be able to take, as an input, arbitrary combinations of molecules and their opacities. The synthetic spectrum computed should be highly customizable, such that it may be readily compared to both photometric and spectroscopic data, often combined in a heterogeneous way across wavelength. To explore such a broad range of parameter space, the numerical implementation of a model (in short, the "code") needs to solve for radiative equilibrium very efficiently and also allow for numerical convergence to be checked in several different ways: number of model layers, spectral resolution of opacity function, number of wavelength bins used, etc. Such a code forms the basis of a flexible radiation package that one may couple to a chemical kinetics code or a 3D general circulation model. The challenges of constructing a 1D radiative-convective model are also discussed in the review article by Marley \& Robinson (2015), where the "convective" part stands for the additional consideration of convective stability, which marks the next step in sophistication of an atmospheric model. 
In the current work, we present a customizable and builtfrom-scratch computer code named $\operatorname{HELIOS}^{6}$, which has or uses the following components.

1. In this initial version, we use the analytical solutions of the radiative transfer equation in the two-stream approximation, as derived by Heng et al. (2014). These solutions enable us to iteratively and self-consistently solve for the temperature-pressure profile of the atmosphere via iteration with its opacity function, which generally depends on temperature, pressure, and wavelength. The synthetic spectrum is obtained as a natural by-product of this self-consistent calculation.

2. For the opacity function of the atmosphere, we use our open-source and custom opacity calculator, HELIOS-K, which was previously published by Grimm \& Heng (2015). The finest resolution we have used is $10^{-5} \mathrm{~cm}^{-1}$ across the entire wavenumber range considered. We then compute $k$-distribution tables from this finely spaced grid of opacities across temperature, pressure, and molecular species.

3. Throughout this work, we assume chemical equilibrium, which effectively means that the chemistry is described by only two parameters: the elemental abundances of carbon and oxygen. Given the input values of these elemental abundances, we then use the validated analytical formulae of Heng \& Lyons (2016) and Heng \& Tsai (2016) to calculate the mixing ratios (abundances normalized to that of molecular hydrogen) of the various molecules. We consider water $\left(\mathrm{H}_{2} \mathrm{O}\right)$, carbon monoxide (CO), carbon dioxide $\left(\mathrm{CO}_{2}\right)$, and methane $\left(\mathrm{CH}_{4}\right)$.

4. We have built HELIOS to run on graphics processing units (GPUs) to maximize the computational throughput. A HELIOS calculation with 101 model layers and 300 wavelength bins takes only a few minutes to complete on a personal computer with an NVIDIA GeForce 750M GPU. ${ }^{7}$ This level of efficiency allows us to effectively perform parameter studies.

In Section 2, we provide a detailed description of our methodology, including the equations and boundary conditions used, the numerical methods, the structure of our grid, the opacity calculations, the chemistry model, and the stellar models used. In Section 3, we subject HELIOS to various tests, and use it to address several lingering ambiguities ${ }^{8}$ in the literature and also to examine six case studies of hot Jupiters. In Section 4, we summarize our results, compare them to previous work, and discuss opportunities for future work.

\section{METHODOLOGY}

\subsection{Radiative Transfer Scheme}

\subsubsection{Preamble}

Any scheme to represent the propagation of radiation through an atmosphere has to solve the radiative transfer

\footnotetext{
6 Named after the Greek god of the Sun.

7 Note that these are fully converged and self-consistent models, which require iteration to solve for radiative equilibrium.

8 We describe these issues as "lingering," because studies in the published literature typically omit the details involved, which prevents us from directly comparing our results to them.
}

equation (Chandrasekhar 1960; Mihalas 1970),

$$
\mu \frac{\partial I_{\lambda}}{\partial \tau_{\lambda}}=I_{\lambda}-S_{\lambda},
$$

where $I_{\lambda}$ is the monochromatic and wavelength-dependent intensity, $\mu \equiv \cos \theta$ is the cosine of the incident angle $(\theta)$ relative to the normal, and $\tau_{\lambda}$ is the optical depth measured from the top of the atmosphere (TOA) downward. We denote the wavelength by $\lambda$. The crucial "length" to adopt in radiative transfer is the optical depth. (Only a nonvanishing $\Delta \tau_{\lambda}$ leads to a change in intensity $\Delta I_{\lambda}$.) The source function $S_{\lambda}$ accounts for both radiation scattered into the line of sight and the thermal emission associated with each location in the medium. Equation (1) is generally difficult to solve, because it is a partial differential equation in $\tau_{\lambda}$ and $\mu$.

A commonly used simplification is to reduce Equation (1) to an ordinary differential equation in $\tau_{\lambda}$ by integrating over the incoming $(-\pi / 2 \leqslant \theta \leqslant 0$ or $-1 \leqslant \mu \leqslant 0)$ and outgoing $(0 \leqslant \theta \leqslant \pi / 2$ or $0 \leqslant \mu \leqslant 1)$ hemispheres and assuming that the ratios of various moments of the intensity are constant and take on specific values. This is known as the "two-stream approximation" (Meador \& Weaver 1980). One may then solve the ordinary differential equation analytically to obtain solutions for pairs of model atmospheric layers (Heng et al. 2014). The moments of the intensity are related by the so-called "Eddington coefficients." Of particular interest to us is the first Eddington coefficient (Heng et al. 2014),

$$
\epsilon=\frac{1}{\mathcal{D}}
$$

which is related to the "diffusivity factor" $\mathcal{D}$. In the next subsection, we show that $\mathcal{D}$ should take on a value between 1 and 2 depending on the thickness of the atmospheric layers.

In the current study, we use the two-stream solutions previously derived by Heng et al. (2014). We note that these solutions allow for the inclusion of nonisotropic scattering via two functions: the single-scattering albedo $\left(\omega_{0}\right)$ and the scattering asymmetry factor $\left(g_{0}\right)$ (Goody \& Yung 1989; Pierrehumbert 2010). Pure absorption and scattering correspond to $\omega_{0}=0$ and $\omega_{0}=1$, respectively. Forward, backward, and isotropic scattering correspond to $g_{0}=1,-1$, and 0 , respectively. Our formulation allows for $\omega_{0}$ and $g_{0}$ to be specified as functions of wavelength/frequency/wavenumber, temperature, and pressure.

Hereafter, the term "flux" describes a wavelength-dependent quantity. ${ }^{9}$ Integrating the flux over all wavelengths, one obtains the "bolometric flux." We also neglect for readability the subscript $\lambda$ for $\tau$ and $B$.

\subsubsection{Exact Solution of the Radiative Transfer Equation in the Pure Absorption Limit}

As previously shown by Heng et al. (2014, and references therein), the radiative transfer equation has an exact solution in the limit of pure absorption $\left(\omega_{0}=0\right)$. We use a staggered grid (see Section 2.2.1), such that the two-stream solutions are applied to the interfaces of a model layer. We label the interfaces by " 1 " and " 2 " and our convention is to locate interace 2 above interface 1 in altitude. If the layer has only one temperature throughout (i.e., it is isothermal), then the fluxes at

\footnotetext{
Accordingly, the units of the flux $F$ are $[F]=\mathrm{erg} \mathrm{s}^{-1} \mathrm{~cm}^{-3}$.
} 
the interfaces are given by

$$
\begin{aligned}
& F_{2, \uparrow}=\mathcal{T} F_{1, \uparrow}+\pi B_{1}(1-\mathcal{T}), \\
& F_{1, \downarrow}=\mathcal{T} F_{2, \downarrow}+\pi B_{1}(1-\mathcal{T}) .
\end{aligned}
$$

The subscripts $\uparrow$ and $\downarrow$ refer to the outgoing and incoming fluxes, respectively. The blackbody intensity within this layer is given by $B_{1}$.

We can improve upon the isothermal-layer treatment by considering a (linear) temperature gradient within the layer (Toon et al. 1989). If we instead Taylor-expand the Planck function in $\tau$ and retain only the constant and linear terms, we obtain

$$
\begin{aligned}
F_{2, \uparrow}= & \mathcal{T} F_{1, \uparrow}+\pi B_{1}(1-\mathcal{T}) \\
& +\pi B^{\prime}\left\{\frac{2}{3}\left[1-e^{-\Delta \tau}\right]-\Delta \tau\left(1-\frac{\mathcal{T}}{3}\right)\right\}, \\
F_{1, \downarrow}= & \mathcal{T} F_{2, \downarrow}+\pi B_{2}(1-\mathcal{T}), \\
& +\pi B^{\prime}\left\{-\frac{2}{3}\left[1-e^{-\Delta \tau}\right]+\Delta \tau\left(1-\frac{\mathcal{T}}{3}\right)\right\} .
\end{aligned}
$$

following the derivation in Heng et al. (2014). The difference in optical depth between the layers is given by $\Delta \tau \equiv \tau_{2}-\tau_{1}$. The gradient of the Planck function is approximated by

$$
B^{\prime} \approx \frac{B_{2}-B_{1}}{\tau_{2}-\tau_{1}},
$$

where $B_{1}$ and $B_{2}$ are now the Planck functions for the temperatures at the interfaces 1 and 2 , respectively.

In both the isothermal and nonisothermal cases, the transmission function or transmissivity is

$$
\begin{aligned}
\mathcal{T} & =2 \int_{0}^{1} \mu \exp \left(-\frac{\Delta \tau}{\mu}\right) d \mu, \\
& =(1-\Delta \tau) \exp (-\Delta \tau)+\Delta \tau^{2} \mathcal{E}_{1}(\Delta \tau),
\end{aligned}
$$

where $\mathcal{E}_{1}$ is the exponential integral of the first order. Unlike for the two-stream solutions, there is no need to specify $\mathcal{D}$ as an input, because it has an exact solution,

$$
\mathcal{D}=-\frac{1}{\Delta \tau} \ln \left[(1-\Delta \tau) \exp (-\Delta \tau)+\Delta \tau^{2} \mathcal{E}_{1}(\Delta \tau)\right]
$$

For very thin layers $(\Delta \tau \ll 1), \mathcal{D}=2$ is an accurate approximation, but as the layer becomes optically thick the value of $\mathcal{D}$ approaches unity (Figure 1). Operationally, since we pick our model grid to be equally spaced in the logarithm of pressure, this means that the value of $\Delta \tau$ is small near the top of the model atmosphere and gradually becomes large (and exceeds unity) at high pressures. Within the context of the twostream approximation, assuming $\mathcal{D}$ to be constant is equivalent to picking a representative or mean value, over the entire atmosphere, of the diffusivity factor.

As already pointed out by Heng et al. (2014), the analytical expression for $\mathcal{D}$ when scattering is present (equivalent to Equation (7)) is unknown.

It is worth emphasizing that Equations (3) and (4) are exact solutions and that the two-stream approximation is not taken. In Section 3.1.5, we compare these exact solutions to the twostream solutions to derive the value of $\mathcal{D}$.

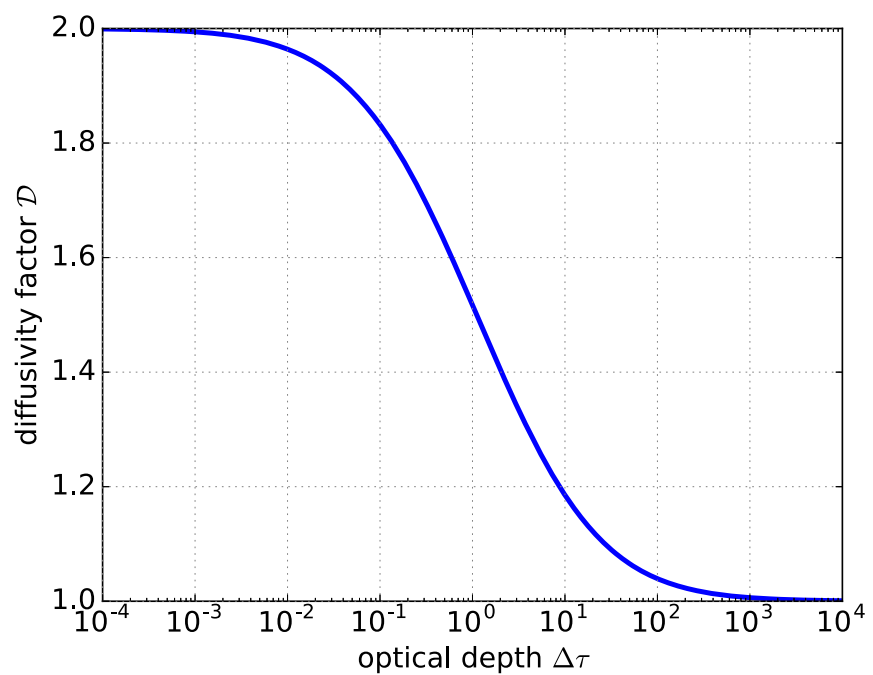

Figure 1. Diffusivity factor $\mathcal{D}$, as a function of the difference in optical depth $\Delta \tau$ across a layer, in the limit of pure absorption.

\subsubsection{Different Flavors of Two-stream Solutions}

We now rederive the two-stream solutions of Heng et al. (2014) without setting $\mathcal{D}=2$, so as to facilitate comparisons with the exact solutions. For all of the solutions presented in this subsection, the transmission function is

$$
\mathcal{T} \equiv \exp \left[-\mathcal{D} \sqrt{\left(1-\omega_{0} g_{0}\right)\left(1-\omega_{0}\right)} \Delta \tau\right]
$$

The simplest two-stream solutions are derived in the limit of pure absorption and isothermal atmospheric layers,

$$
\begin{aligned}
& F_{2, \uparrow}=\mathcal{T} F_{1, \uparrow}+2 \pi \epsilon B_{1}(1-\mathcal{T}), \\
& F_{1, \downarrow}=\mathcal{T} F_{2, \downarrow}+2 \pi \epsilon B_{1}(1-\mathcal{T}) .
\end{aligned}
$$

Without scattering $\left(\omega_{0}=0\right)$, the coupling coefficients are $\zeta_{+}=1$ and $\zeta_{-}=0$, and the transmission function simply becomes $\mathcal{T}=\exp (-\mathcal{D} \Delta \tau)$. If we increase the sophistication of the model by considering nonisothermal layers and pure absorption, we obtain

$$
\begin{aligned}
& F_{2, \uparrow}=\mathcal{T} F_{1, \uparrow}+2 \pi \epsilon\left[B_{1}-\mathcal{T} B_{2}+\epsilon B^{\prime}(1-\mathcal{T})\right], \\
& F_{1, \downarrow}=\mathcal{T} F_{2, \downarrow}+2 \pi \epsilon\left[B_{2}-\mathcal{T} B_{1}-\epsilon B^{\prime}(1-\mathcal{T})\right] .
\end{aligned}
$$

For isothermal atmospheric layers with nonisotropic scattering included, the two-stream solutions for the fluxes read

$$
\begin{aligned}
& F_{2, \uparrow}=\frac{1}{\alpha}\left[\xi F_{1, \uparrow}-\beta F_{2, \downarrow}+2 \pi \epsilon B_{1}(\beta-v)\right], \\
& F_{1, \downarrow}=\frac{1}{\alpha}\left[\xi F_{2, \downarrow}-\beta F_{1, \uparrow}+2 \pi \epsilon B_{1}(\beta-v)\right] .
\end{aligned}
$$

The coefficients $\alpha, \beta, \xi, v$ are defined as

$$
\begin{aligned}
\alpha & \equiv \zeta_{-}^{2} \mathcal{T}^{2}-\zeta_{+}^{2}, \\
\beta & \equiv \zeta_{+} \zeta_{-}\left(1-\mathcal{T}^{2}\right), \\
\xi & \equiv\left(\zeta_{-}^{2}-\zeta_{+}^{2}\right) \mathcal{T}, \\
v & \equiv\left(\zeta_{-}^{2} \mathcal{T}+\zeta_{+}^{2}\right)(1-\mathcal{T}),
\end{aligned}
$$


with the coupling coefficients being

$$
\zeta_{ \pm} \equiv \frac{1}{2}\left[1 \pm\left(\frac{1-\omega_{0}}{1-\omega_{0} g_{0}}\right)^{1 / 2}\right]
$$

In the limit of $\omega_{0}=1$, the equations in (11) are replaced by

$$
\begin{aligned}
& F_{2, \uparrow}=F_{1, \uparrow}-\frac{\mathcal{D}\left(1-g_{0}\right) \tau_{0}\left(F_{1, \uparrow}-F_{2, \downarrow}\right)}{2+\mathcal{D}\left(1-g_{0}\right) \tau_{0}}, \\
& F_{1, \downarrow}=F_{2, \downarrow}+\frac{\mathcal{D}\left(1-g_{0}\right) \tau_{0}\left(F_{1, \uparrow}-F_{2, \downarrow}\right)}{2+\mathcal{D}\left(1-g_{0}\right) \tau_{0}} .
\end{aligned}
$$

These solutions give the correct limits of a transparent or opaque atmosphere when $\omega_{0}=1$ (Heng et al. 2014). The general solutions stated before in Equation (11) do not reproduce this limit.

Our most sophisticated two-stream solutions include nonisotropic scattering and nonisothermal model atmospheric layers,

$$
\begin{aligned}
F_{2, \uparrow}= & \frac{1}{\alpha}\left\{\xi F_{1, \uparrow}-\beta F_{2, \downarrow}+2 \pi \epsilon\left[B_{1}(\alpha+\beta)-B_{2} \xi\right.\right. \\
& \left.\left.+\frac{\epsilon}{1+\omega_{0} g_{0}} B^{\prime}(\alpha-\xi-\beta)\right]\right\}, \\
F_{1, \downarrow}= & \frac{1}{\alpha}\left\{\xi F_{2, \downarrow}-\beta F_{1, \uparrow}+2 \pi \epsilon\left[B_{2}(\alpha+\beta)-B_{1} \xi\right.\right. \\
& \left.\left.+\frac{\epsilon}{1+\omega_{0} g_{0}} B^{\prime}(\xi-\alpha+\beta)\right]\right\} .
\end{aligned}
$$

Note that in the nonisothermal approach a single constant gradient of $B^{\prime}$ is assumed within a layer. Thus $B_{1}$ and $B_{2}$ are placed at the interfaces. ${ }^{10}$ The coefficients $\alpha, \beta$, and $\xi$, as well as the coupling coefficients $\zeta_{ \pm}$, retain the same functional forms as in the case with isothermal layers.

Generally, we find that the nonisothermal solutions attain more rapid numerical convergence (to radiative equilibrium). In principle, if a large enough number of isothermal layers are used, the isothermal and nonisothermal calculations should agree.

\subsubsection{Rayleigh Scattering}

To include the effects of Rayleigh scattering by molecules, we use the cross section (Sneep \& Ubachs 2005),

$$
\sigma_{\text {scat }, \lambda}=\frac{24 \pi^{3}}{n_{\text {ref }}^{2} \lambda^{4}}\left(\frac{n_{\lambda}^{2}-1}{n_{\lambda}^{2}+2}\right)^{2} K_{\lambda},
$$

where $n_{\text {ref }}$ is the number density at a reference temperature and pressure, $n_{\lambda}$ is the wavelength-dependent refractive index, and $K_{\lambda}$ is the King factor, which is a correction factor for polarization.

In the current study, we focus on Rayleigh scattering by hydrogen molecules, but our approach may be straightforwardly generalized to other molecules. We ignore the contribution due to helium, which is less than $1 \%$ of that due to molecular hydrogen. For $\mathrm{H}_{2}$, we use $n_{\text {ref }}=2.68678 \times 10^{19} \mathrm{~cm}^{-3}, K=1$,

\footnotetext{
10 In practice, in the numerical implementation of the equations one layer has to be divided into two sublayers (see Section 2.2.1).
}

and

$$
n_{\lambda}=13.58 \times 10^{-5}\left(1+7.52 \times 10^{-11} \mathrm{~cm}^{2} \lambda^{-2}\right)+1 .
$$

The influence of Rayleigh scattering enters via its inclusion, as $\sigma_{\text {scat }, \lambda} / \bar{m}$, in the opacity of each model layer, where $\bar{m}$ is the mean molecular mass, and also via the single-scattering albedo $\omega_{0}$. The dashed line in Figure 3 shows the opacity of Rayleigh scattering by $\mathrm{H}_{2}$, which dominates in the optical but becomes subdominant, compared to molecular absorption, in the infrared due to its dropoff with $\lambda^{-4}$.

If the scattering dominates and $\left(1-\omega_{0}\right)<10^{-6}$ in this layer and waveband, then we switch to the pure scattering solutions (Equation (14)).

\subsection{Numerical Method}

\subsubsection{Model Grid}

For the isothermal treatment, a staggered grid is used with the layers being separated by interfaces. There are $n$ layers and $n+1$ interfaces. The grid is evenly spaced in height or the logarithm of pressure, which serves as the vertical coordinate. The thickness of the $i$ th layer is given by

$$
\Delta z_{i}=\frac{k_{\mathrm{B}} T_{i}}{\bar{m} g} \ln \left(\frac{P_{i, \text { inter }}}{P_{i+1, \text { inter }}}\right),
$$

with $k_{\mathrm{B}}$ being the Boltzmann constant and $g$ the surface gravity. For hydrogen-dominated atmospheres, we set $\bar{m}=2.4 m_{p}$ with $m_{p}$ being the mass of the proton. The pressures at the interfaces are represented by $P_{i, \text { inter }}$ and $P_{i+1, \text { inter }}$. The preceding expression is obtained from integrating the equation of hydrostatic balance over a model layer and assuming isothermality and the equation of state for an ideal gas.

The contribution to the optical depth ${ }^{11}$ from the $i$ th layer is

$$
\Delta \tau_{i}=\Delta m_{\mathrm{col}, i} \kappa_{i}=\frac{P_{i, \text { inter }}-P_{i+1, \text { inter }}}{g} \kappa_{i},
$$

where $\kappa_{i}$ is the opacity and $\Delta m_{\mathrm{col}, i}$ is the difference in column mass, which can be further written in terms of pressure and surface gravity.

For the nonisothermal grid, we require a more sophisticated grid layout, which is shown in Figure 2. Each layer has a temperature and pressure, located at its center. To compute the fluxes, we need to interpolate across the temperature and pressure grids to obtain their values at the interfaces. A key quantity to compute is the Planck function $B$, which relates the temperature to the thermal emission of a layer. If one constructs the grid using a single gradient $B^{\prime}$ of the Planck function over the whole layer, one is essentially decoupling the radiative transfer process from the temperature at the center of the layer. We solve this problem by splitting each layer into two sublayers, leading to two $B^{\prime}$ values within a layer. The fluxes are propagated first from the lower interface to the layer center, then from the layer center to the upper interface (and vice versa), similar to the approach taken in, e.g., Mendonça et al. (2015). In this manner, both the layer centers and interfaces are involved in the iteration for radiative equilibrium.

\footnotetext{
${ }^{11}$ To be pedantic, the optical depth is a coordinate. It is the difference in optical depth that is needed for radiative transfer. The analogy is to distance versus displacement.
} 


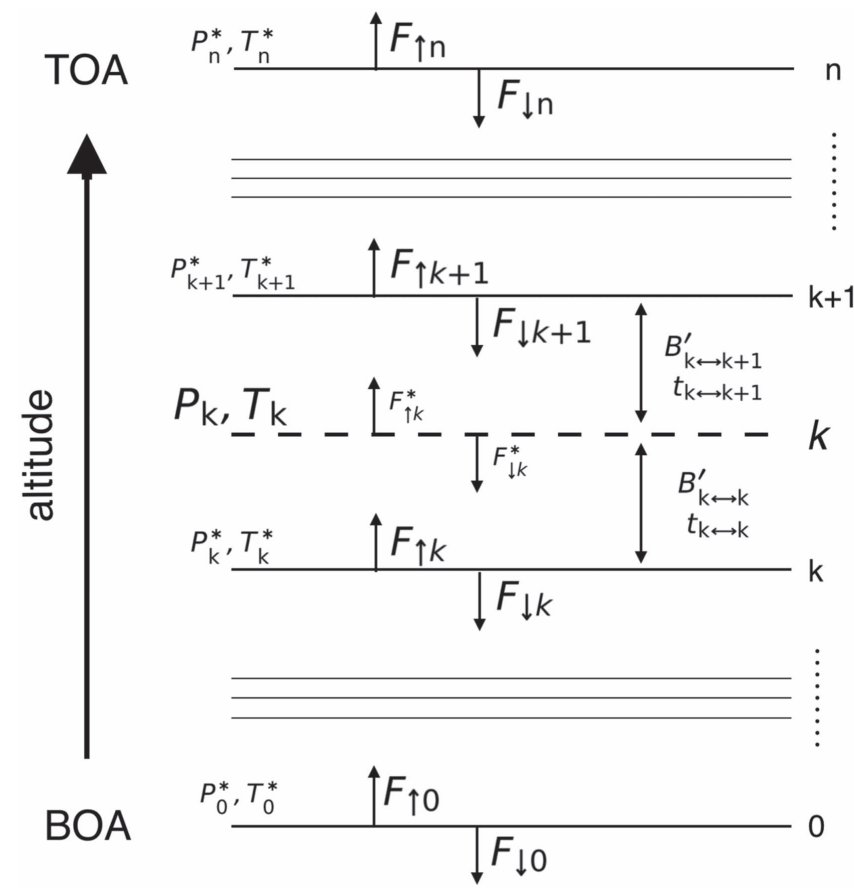

Figure 2. Staggered grid used for models with nonisothermal layers. The boundary conditions are applied at the top (stellar irradiation) and bottom (internal heat flux) of the model atmosphere, which are also the $n$th and 0th interfaces, respectively. The pressure and temperature are located at the center of each layer, while the fluxes traversing a layer are computed at the layer interfaces. We further divide each layer into two sublayers during the iteration for radiative equilibrium (see text for details). In the schematic, we have focused on the $k$ th layer and the various quantities associated with its center and interfaces. Quantities marked with an asterisk are temporarily used in the computation, but not stored as the final output. The layers are evenly spaced in the logarithm of pressure.

Finally, in the nonisothermal grid, a numerical caveat arises in the upper atmosphere. There, the difference in optical depth $\Delta \tau_{i}$ of a layer $i$ is tiny (due to the very small pressure) and thus the denominator of Equation (5) vanishes, which in turn leads to numerical issues for $B^{\prime}$ in Equation (15). To prevent this, we keep the sublayered grid of the nonisothermal approach, but switch in each sublayer from the nonisothermal prescription (Equation (15)) to the isothermal one (Equation (11)) whenever $\Delta \tau_{i}<10^{-4}$.

\subsubsection{Boundary Conditions}

At the TOA, which is also the $n$th interface of the model atmosphere, the flux is given by

$$
F_{n, \downarrow}=f\left(\frac{R_{\star}}{a}\right)^{2} \pi B_{\star}
$$

where $R_{\star}$ is the stellar radius, $a$ is the orbital distance of the planet, and $B_{\star}$ is the stellar blackbody function. This represents the heating from the incident stellar flux. Most of the quantities in the preceding expression are astronomical observables (or quantities that may be inferred from the observations). It is possible to replace $B_{\star}$ by a more sophisticated model of the stellar spectrum (see Section 2.5).

The quantity $f$ is a parameter that describes the redistribution of heat from the dayside to the nightside of a tidally locked hot Jupiter, which is dictated by an interplay between atmospheric dynamics and radiative cooling. In principle, its value may be inferred from infrared phase curves. Theoretically, it is bounded between $f=1 / 4$ (full redistribution) and $f=1$ (no redistribution). Since we are using our 1D, plane-parallel model to describe the dayside emission spectra of hot Jupiters, the value of $f$ is a proxy for the dayside-integrated absorption and re-emission of radiation. In the current study, we adopt $f=2 / 3$ following the arguments in, e.g., Burrows et al. (2008) and Spiegel \& Burrows (2010).

At the bottom of the model atmosphere (BOA), we have included the option to specify an internal radiative heat flux $\left(\pi B_{\text {intern }}\right)$, such that

$$
\int \pi B_{\text {intern }} d \lambda=\sigma_{\mathrm{SB}} T_{\text {intern }}^{4},
$$

where $\lambda$ is the wavelength, $\sigma_{\mathrm{SB}}$ is the Stefan-Boltzmann constant, $T_{\text {intern }}$ is the internal temperature, $B_{\text {intern }} \equiv B\left(T_{\text {intern }}\right)$, and $B$ is the Planck function. The internal heat flux reflects the thermal heating due to gravitational contraction. The BOA is also the 0th interface. It is important to note that any form of atmospheric heating is associated with the net flux (the difference between the outgoing and incoming fluxes) (Heng et al. 2014),

$$
\pi B_{\text {intern }}=F_{0, \uparrow}-F_{0, \downarrow} .
$$

In our current study, we set $T_{\text {intern }}=0 \mathrm{~K}$ in the absence of such constraints on hot Jupiters.

\subsubsection{Iterating for Radiative Equilibrium}

Within each model layer of the atmosphere, its temperature and pressure determine its absorption and scattering properties, given by molecular abundances and opacities, which in turn determine the transmission function and fluxes. However, as flux enters and exits the layer, the temperature changes, which in turn changes the opacity. Clearly, this is an iterative process. It turns out that one is iterating for radiative equilibrium, which is a statement of local energy conservation (Heng et al. 2014). Local energy conservation implies global energy conservation, but not vice versa (Heng \& Lyons 2016).

We integrate the fluxes $\left(F_{\uparrow}\right.$ and $\left.F_{\downarrow}\right)$ over the entire spectral range to obtain the bolometric fluxes $\left(\mathcal{F}_{\uparrow}\right.$ and $\left.\mathcal{F}_{\downarrow}\right)$, which in turn allows us to construct the bolometric net flux $\left(\mathcal{F}_{-} \equiv \mathcal{F}_{\uparrow}-\mathcal{F}_{\downarrow}\right)$. For the $i$ th layer, the divergence ${ }^{12}$ of the bolometric net flux becomes

$$
\frac{\Delta \mathcal{F}_{i,-}}{\Delta z_{i}}=\frac{\left(\mathcal{F}_{i+1, \uparrow}-\mathcal{F}_{i+1, \downarrow}\right)-\left(\mathcal{F}_{i, \uparrow}-\mathcal{F}_{i, \downarrow}\right)}{\Delta z_{i}} .
$$

Between successive timesteps, the change in temperature of the $i$ th layer then becomes (Heng et al. 2014)

$$
\Delta T_{i}=\frac{1}{\rho_{i} c_{\mathrm{p}}} \frac{\Delta \mathcal{F}_{i,-}}{\Delta z_{i}} \Delta t_{i}
$$

where $\rho_{i}$ is the local density and $\Delta t_{i}$ is the numerical timestep. The specific heat capacity of an ideal gas at constant pressure is (Pierrehumbert 2010)

$$
c_{\mathrm{p}}=\frac{2+n_{\mathrm{dof}}}{2 \bar{m}} k_{\mathrm{B}},
$$

\footnotetext{
${ }^{12}$ In $1 \mathrm{D}$, the divergence is simply the vertical gradient.
} 
where we set the number of degrees of freedom of the gas to be $n_{\text {dof }}=5$, as is valid for a diatomic molecule (ignoring the vibrational modes) like molecular hydrogen, the main component of the atmospheres of gas planets. This simplification does not hold should the atmospheric composition change, e.g., by dissociation of molecular hydrogen at very high temperatures. Since the only occurrence of $c_{\mathrm{p}}$ in our model is in the timestepping algorithm, this flaw is of only minor concern for our cause, but it would render, e.g., the calculation of the entropy inaccurate.

In practice, we start with an arbitrary temperature profile with $\Delta \mathcal{F}_{i,-} \neq 0$. We perform the iteration described until $\Delta \mathcal{F}_{i,-}$ vanishes for each layer, which is our numerical condition for radiative equilibrium (compare with Equation (29)). Physically, each atmospheric layer emits the same amount of energy as it receives.

When scattering is present, the flux solutions become coupled. Each array of outgoing or incoming fluxes cannot be populated independently of the others. This is solved iteratively by starting with the coupled dependences as zero and populating the flux arrays multiple times in each temperature step. We include four additional scattering iterations in the full radiative transfer calculation because the flux values are still known from the previous timestep, and 80 scattering iterations for pure post-processing purposes (propagating only once through the atmosphere).

\subsubsection{Numerical Timestepping}

For the numerical timestepping, there are two options in HELIOS. The first option uses a fixed and uniform timestep $(\Delta t)$ for every model layer. Typically, we choose $10^{2} \lesssim \Delta t \lesssim 10^{4} \mathrm{~s}$. The challenge is that $\Delta T \propto \rho^{-1}$ and $\rho$ may vary by several orders of magnitude across our model atmosphere. With a uniform timestep, the upper layers of the atmosphere attain convergence much more rapidly than the lower layers. Thus, this approach is plausible and technically correct, but infeasible.

A more efficient approach is to implement an adaptive timestepping scheme that uses a different timestep for each model layer. Specifically, the timestep in the $i$ th layer is related to the radiative timescale $\left(t_{i, \text { rad }}\right)$,

$$
\Delta t_{i}=f_{i, \mathrm{pre}} t_{i, \mathrm{rad}},
$$

where $f_{i \text {,pre }}$ is a prefactor to adjust to the optimal value of $\Delta t_{i}$. The radiative timescale is approximated by

$$
t_{i, \text { rad }} \approx \frac{c_{\mathrm{p}} P_{i}}{\sigma_{\mathrm{SB}} g T_{i}^{3}},
$$

where the temperature and pressure of the $i$ th layer are given by $T_{i}$ and $P_{i}$, respectively. With this improved timestepping scheme, the timestep becomes larger as one goes deeper into the model atmosphere. The evolution of the model does not strictly correspond to a physical evolution, but is rather a convenient way of reaching a numerical steady state.

To further optimize the efficiency of HELIOS, we also allow the timestep to vary in time as the model approaches radiative equilibrium. Specifically, the algorithm checks in each layer whether the temperature has oscillated for the most recent six successive timesteps. We find oscillations in temperature to be a robust and practical indicator of having adopted too large a
Table 1

Opacity Sources Used in this Work

\begin{tabular}{ll}
\hline \hline Name & Source \\
\hline $\mathrm{H}_{2} \mathrm{O}$ & HITEMP database $^{\mathrm{a}}$ (Rothman et al. 2010) $_{\mathrm{CO}_{2}}$ \\
$\mathrm{CO}$ & HITEMP database $^{\mathrm{a}}$ \\
$\mathrm{CH}_{4}$ & HITEMP database $^{\mathrm{a}}$ \\
$\mathrm{CIA}$ & HITRAN database \\
Rayleigh scattering & HITRAN CIA database (Richard et al. 2012) \\
\hline
\end{tabular}

Notes.

a hitran.org/hitemp/

${ }^{\mathrm{b}}$ www.cfa.harvard.edu/hitran/

timestep. If oscillations are detected, the timestep is reduced by $33 \%$. By contrast, if no oscillations are detected (i.e., the change in temperature is monotonic), then the timestep is increased by $10 \%$.

We note that the purpose of the prefactor $\left(f_{i, \text { pre }}\right)$ is to dampen sudden spikes in $\Delta \mathcal{F}_{i,-}$. For practical purposes, it takes the form of

$$
f_{i, \text { pre }}=\frac{10^{5}}{\left[\left|\Delta \mathcal{F}_{i,-}\right| /\left(\mathrm{erg} \mathrm{s}^{-1} \mathrm{~cm}^{-2}\right)\right]^{0.9}},
$$

which leads the temperature iteration step $\Delta T_{i}$ to depend only on $\Delta \mathcal{F}_{i,-}^{0.1}$, which guarantees the correct direction of the evolution but substantially smoothes irregularities, making the iteration process significantly more stable.

Finally, we need a condition to judge whether radiative equilibrium has been established. Usually, one would assume a criterion demanding the rate of temperature change to be below a certain threshold, $\Delta T / \Delta t<\delta_{\text {limit }}$, and evaluate whether this is satisfied in every layer. However, if $\Delta t$ is variable and not representing a physical time, then the utility of this approach becomes suspect. Instead of setting a threshold on the consequence of radiative equilibrium (changes in temperature), we set one on its cause (a vanishing bolometric flux divergence). We use the dimensionless convergence criterion,

$$
\frac{\Delta \mathcal{F}_{-}}{\sigma_{\mathrm{SB}} T^{4}}<10^{-7}
$$

where the change in bolometric net flux is normalized by the thermal emission associated with each layer. In practice, this criterion results in changes in temperature of less than $4 \mathrm{~K}$ at the BOA and less than $1 \mathrm{~K}$ in the photospheric regions, which impacts the emission spectrum by less than $0.5 \%$.

\subsection{Calculating Opacities and Transmission Functions}

Our method for computing the opacities (cross sections per unit mass) of molecules has previously been elucidated in Grimm \& Heng (2015), who published an opacity calculator named HELIOS-K that is part of the HELIOS radiation package. As such, we do not repeat the detailed explanations of Grimm \& Heng (2015) and instead highlight only the salient points. We include the opacities associated with the four main infrared absorbers: $\mathrm{H}_{2} \mathrm{O}, \mathrm{CO}_{2}, \mathrm{CO}$ and $\mathrm{CH}_{4}$. We also include the opacities associated with the collision-induced absorption (CIA) of $\mathrm{H}_{2}-\mathrm{H}_{2}$ and $\mathrm{H}_{2}-\mathrm{He}$ pairs. Table 1 states the spectroscopic line lists used to compute our opacities, while 


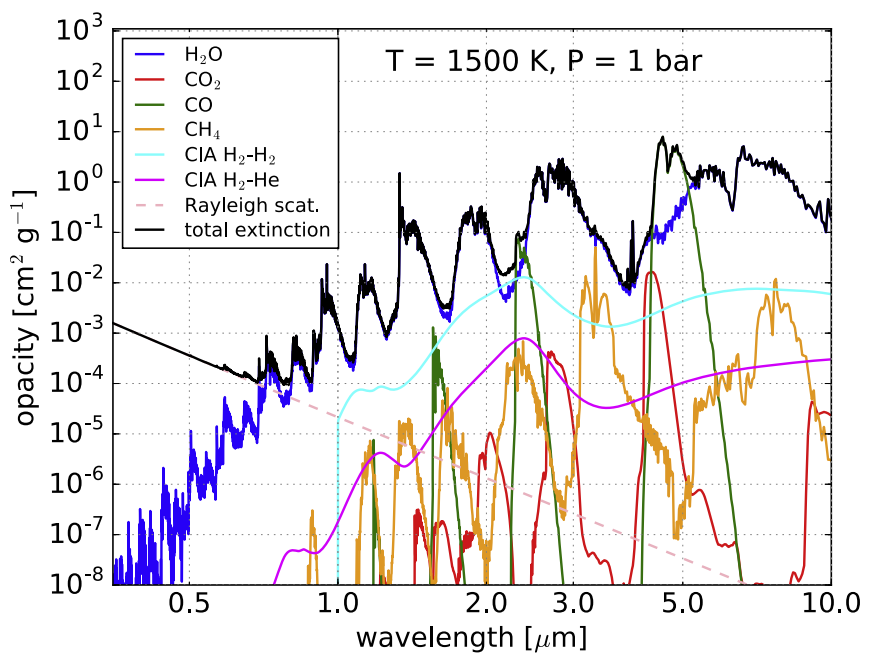

Figure 3. Opacities, as functions of wavelength, for all of the opacity sources used in the current study, computed using HELIOS-K (Grimm \& Heng 2015). For illustration, we set $T=1500 \mathrm{~K}$ and $P=1$ bar. Each opacity is weighted by its mass mixing ratio. We include only Rayleigh scattering by molecular hydrogen, but CIA associated with both $\mathrm{H}_{2}-\mathrm{H}_{2}$ and $\mathrm{H}_{2}-\mathrm{He}$ pairs.

Figure 3 displays the final weighted opacities used in the code at one temperature and pressure. ${ }^{13}$

The first step involves calculating the opacity function (cross section per unit mass as a function of wavelength, temperature, and pressure), which includes all of the molecules previously mentioned, at a given spectral resolution. If the spectral resolution is too coarse, then spectral lines may be missed or omitted, which leads to an underestimation of the true opacity. To avoid this pitfall, we use a resolution of $10^{-5} \mathrm{~cm}^{-1}$. Since the wavenumber range goes up to $\sim 10^{4} \mathrm{~cm}^{-1}$, this means that we are sampling the opacity function at $\sim 10^{9}$ points, which approaches a true line-by-line calculation.

The shape of each spectral line is described by a Voigt profile. A major uncertainty associated with this approach, which remains an unsolved physics problem, is that the far line wings of the Voigt profile overestimate or underestimate the true opacity contribution depending on the molecule (see Grimm \& Heng 2015 for a discussion). The common practice is to truncate each Voigt profile at some fixed spectral width. For example, Sharp \& Burrows (2007) use a line-wing cutoff of $\min (25 P / 1 \mathrm{~atm}, 100) \mathrm{cm}^{-1}$. We use a cutoff of $100 \mathrm{~cm}^{-1}$ except for water, where we instead use $25 \mathrm{~cm}^{-1}$. We emphasize that the correct functional form of these far line wings is unknown.

To speed up our calculations, we wish to avoid having to deal with integrating over $\sim 10^{9}$ points in the opacity function to obtain the transmissivities. Instead, we employ the $k$ distribution method to calculate the transmission function within each wavelength bin,

$$
\mathcal{T}=\int_{0}^{1} \psi d y
$$

where the integrand, which is given by $\psi \equiv \exp (-\mathcal{D} \Delta \tau)$, is a function of a new variable $(y)$ that is bounded between 0 and 1. We refer the reader to Grimm \& Heng (2015) for a detailed

\footnotetext{
13 The reader should be aware that, in this first version of HELIOS, we omit greenhouse gases like $\mathrm{NH}_{3}, \mathrm{HCN}, \mathrm{C}_{2} \mathrm{H}_{2}$, and the alkali metals $\mathrm{Na}$ and $\mathrm{K}$, which may have an impact on the atmospheric structure. $\mathrm{H}$ and $\mathrm{H}^{-}$absorption may also be important at high temperatures. Nevertheless, our starting set of four molecules is sufficient for us to build up the first version of a radiative transfer code, and we intend to augment this set in the future.
}

explanation of the $k$-distribution method and instead focus on our method for numerically evaluating the preceding integral, which we solve by applying the Gauss-Legendre quadrature rule,

$$
\int_{0}^{1} \psi d y=\frac{1}{2} \sum_{g=1}^{20} w_{g} \psi\left(\frac{1+y_{g}}{2}\right),
$$

where $y_{g}$ is the $g$ th root of the 20th order Legendre polynomial $P_{20}$. The corresponding Gaussian weight $w_{g}$ is (Abramowitz \& Stegun 1972)

$$
w_{g}=\frac{2}{\left[1-y_{g}^{2}\right] P_{20}^{\prime}\left[y_{g}\right]^{2}},
$$

with $P_{20}^{\prime}$ being the derivative of $P_{20}$. We find that using a 20th order Gaussian quadrature rule is sufficient by comparing our calculations to direct integration using Simpson's rule (not shown).

The obvious advantage of using Gaussian quadrature over direct integration is the enhanced computational efficiency. In HELIOS, we propagate the fluxes through the model atmosphere for each of the 20 Gaussian points and perform the Gaussian quadrature sum at the end of the propagation to obtain the flux associated with a wavelength bin. Since the fluxes follow inhomogeneous paths across pairs of layers (i.e., the temperatures and pressures are not constant along these paths) and we also add the $k$-distribution functions of the various molecules, we have to invoke the correlated- $k$ approximation twice (Grimm \& Heng 2015).

Computing the flux through each Gaussian point is equivalent to expressing the transmission function through layer $i$ and waveband $l$ by

$$
\mathcal{T}_{i, l}=\sum_{g=1}^{20} w_{g} e^{-\kappa_{i, l, g} \Delta m_{\mathrm{col}, i},}
$$

which is nothing other than a discrete form of Equation (30) applied to our model. The $g$ th $k$-coefficient in waveband $l$ is written as

$$
\kappa_{i, l, g}=\sum_{j=1}^{6} \mathcal{X}_{j}\left(T_{i}, P_{i}\right) \kappa_{j, l, g}\left(T_{i}, P_{i}\right),
$$

where $T_{i}$ and $P_{i}$ are the temperature and pressure at the center of the $i$ th layer in the isothermal layer grid and also at the interfaces in the nonisothermal layer grid where we have sublayers. In the latter case, we calculate the opacity in the center and at the interface and take their average to obtain the value in the connecting sublayer. The mixing ratios and opacities are generally functions of temperature and pressure. At this point, we have to distinguish between the mixing ratios by volume $\left(X_{j}\right)$ and the mixing ratios by mass $\left(\mathcal{X}_{j}\right)$. The chemistry formulae (see Section 2.4) are constructed to compute $X_{j}$. However, to construct $\kappa_{i}$ we need

$$
\mathcal{X}_{j}=\frac{X_{j} m_{j}}{\bar{m}}
$$

where $m_{j}$ is the mass of the $j$ th molecule.

In Equations (34) and (35), the indices $j=1,2,3,4$ refer to the four molecules being included in the current study: $\mathrm{CO}$, $\mathrm{CO}_{2}, \mathrm{H}_{2} \mathrm{O}$, and $\mathrm{CH}_{4}$. For these molecules, $X_{j}$ is computed using the chemistry model. The indices $j=5$ and $j=6$ refer to the 
CIA opacities associated with $\mathrm{H}_{2}-\mathrm{H}_{2}$ and $\mathrm{H}_{2}-\mathrm{He}$, respectively. For these, we use $X_{5}=1$ and $X_{6}=0.1$ to approximately reflect cosmic abundance. We use $m_{5}=2 m_{p}$ and $m_{6}=4 m_{p}$.

By using Equation (34), we inherently assume the spectral lines of the various molecules to be perfectly correlated. In general, there are three limits: perfectly correlated, randomly overlapping (perfectly uncorrelated), and disjoint lines (see Pierrehumbert 2010 for a review). Real spectral lines behave in a way that is intermediate between these limits. Lacis \& Oinas (1991) and, more recently, Amundsen et al. (2016) have implemented a randomly overlapping method for combining the opacities of the different molecules, which is computationally more expensive because it involves multiple summations. As the spectral resolution increases (and the bin size decreases), these approaches should converge to the same answer. The true accuracy of these approaches remains unquantified in the hot atmosphere regime and needs to be tested by a true line-by-line calculation, where each of the $\gtrsim 10^{9}$ line shapes is numerically resolved. This is the subject of future work and is beyond the scope of the present paper.

In HELIOS, the $k$-coefficients are read in from a fourdimensional, precomputed table in temperature $(100 \leqslant T \leqslant$ $2900 \mathrm{~K}, \Delta T=200 \mathrm{~K})$, pressure $\left(10^{-6} \leqslant P \leqslant 10^{3}\right.$ bar, $\Delta \log _{10}$ $P=0.5),{ }^{14}$ and wavelength $\left(0.33 \leqslant \lambda \leqslant 10^{5} \mu \mathrm{m}\right)$, with the bins subdivided by 20 Gaussian points. The opacities are used at the constructed wavelength (and Gaussian point) values, but are linearly interpolated across $T$ and $\log P$.

Finally, we note that we use 300 wavelength bins (equally spaced in wavenumber) when running HELIOS to solve for radiative equilibrium. Upon obtaining the converged temperature-pressure profile, we then use it to compute synthetic spectra in 3000 wavelength bins as a post-processing step. We find that this approach produces essentially identical results to performing the entire calculation using 3000 wavelength bins (not shown).

\subsection{Chemistry Model}

Given the elemental abundances of carbon $\left(n_{\mathrm{C}}\right)$ and oxygen $\left(n_{\mathrm{O}}\right)$, we would like to compute the mixing ratios (number densities normalized by that of molecular hydrogen) of the four molecules used in our model as functions of temperature and pressure. This requires a chemistry model. To this end, we use the analytical calculations of Heng \& Lyons (2016). Specifically, Heng et al. (2016) laid out the theoretical formalism, which led to the formulae in Equations (12), (20), and (21) in Heng \& Lyons (2016) that we are using. Heng \& Tsai (2016) demonstrated that these formulae are accurate compared to a Gibbs free energy minimization code, even when nitrogen is added to the system. We explicitly demonstrate the agreement between Equations (12), (20), and (21) of Heng \& Lyons (2016) and the calculations from the TEA code of Blecic et al. (2015) in Figure 4. Since we do not study atmospheres with $\mathrm{C} / \mathrm{O}>1$, we ignore $\mathrm{C}_{2} \mathrm{H}_{2}$.

We define "solar elemental abundance" to be $n_{\mathrm{C}}=2.5 \times$ $10^{-4}$ and $n_{\mathrm{O}}=5 \times 10^{-4}$, such that $\mathrm{C} / \mathrm{O} \equiv n_{\mathrm{C}} / n_{\mathrm{O}}=0.5$. In this study, we keep the value of $n_{\mathrm{O}}$ fixed and vary $n_{\mathrm{C}}$ when we vary $\mathrm{C} / \mathrm{O}$. For example, a model with $\mathrm{C} / \mathrm{O}=0.1$ has $n_{\mathrm{C}}=5 \times 10^{-5}$ and $n_{\mathrm{O}}=5 \times 10^{-4}$.

\footnotetext{
14 If the layer pressure or temperature exceeds the range of the values in the table, the opacity is simply taken to correspond to the closest pre-tabulated value.
}

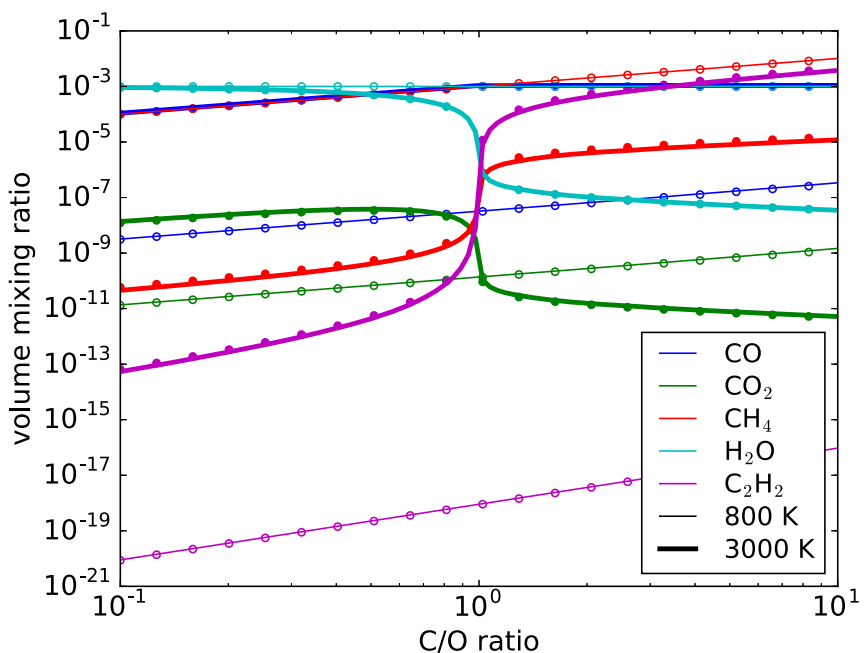

Figure 4. Validation of our analytical chemistry model (Heng \& Lyons 2016; circles) by calculations using the Gibbs free energy minimization code, TEA (Blecic et al. 2015; solid curves). For illustration, we have computed the volume mixing ratios as functions of $\mathrm{C} / \mathrm{O}$ and examined $P=1 \mathrm{bar}$ and $T=800$ and $3000 \mathrm{~K}$.

Following the convention of the astronomers, we refer to the "metallicity" as the set of values of the elemental abundances that have atomic numbers larger than that of helium. In our model, these are $n_{\mathrm{C}}$ and $n_{\mathrm{O}}$. These numbers are simply decreased or increased by a constant factor when the metallicity is varied. For example, a model with $3 \times$ solar metallicity has $n_{\mathrm{C}}=7.5 \times 10^{-4}$ and $n_{\mathrm{O}}=1.5 \times 10^{-3}$, but still retains $\mathrm{C} / \mathrm{O}=0.5$.

Figure 5 shows examples of our calculations of the molecular mixing ratios as functions of temperature, $\mathrm{C} / \mathrm{O}$, and metallicity. To develop some intuition for the relative abundances of molecules present in our model atmospheres, we have included shaded columns indicating the dayside-averaged temperatures of five of the seven exoplanets being studied in the current paper. ${ }^{15}$

\subsection{Stellar Models}

For any atmosphere of the exoplanet irradiated by the host star, one needs a description of the incident stellar flux. The simplest approach is to adopt a Planck function, where the only input is the effective temperature of the stellar photosphere $\left(T_{\star}\right)$. The next level of sophistication requires the use of models such as MARCS, PHOENIX, or Kurucz (ATLAS) that predict the photospheric emission from a star. Specifically in this work, we use the latter two: PHOENIX (Allard \& Hauschildt 1995; Husser et al. 2013) and Kurucz models (Kurucz 1979; Murphy $\&$ Meiksin 2004; Munari et al. 2005). ${ }^{16}$ For completeness, Figure 6 shows the stellar spectra we used to model our sample of six hot Jupiters in Section 3.2. The choice of stellar model has two primary effects. First, since the secondary emission spectrum is the ratio of the exoplanet's to the star's flux, features in the stellar spectrum are imprinted onto it. Second, differences in the stellar spectrum cause changes in the way the

\footnotetext{
15 Two planets are hotter than $3000 \mathrm{~K}$ and not visible in Figure 5. As we have tabulated Gibbs free energies only up to $3000 \mathrm{~K}$, we assume the chemistry to be that at $3000 \mathrm{~K}$ if the temperatures exceed $3000 \mathrm{~K}$.

16 The PHOENIX spectra are downloaded directly from their online library at ftp://phoenix.astro.physik.uni-goettingen.de/HiResFITS/ and interpolated in stellar temperature $T_{\star}$, surface gravity $g_{\star}$, and metallicity to fit the stellar parameters shown in Table 2 . The Kurucz spectra are interpolated in $T_{\star}$ and $g_{\star}$.
} 

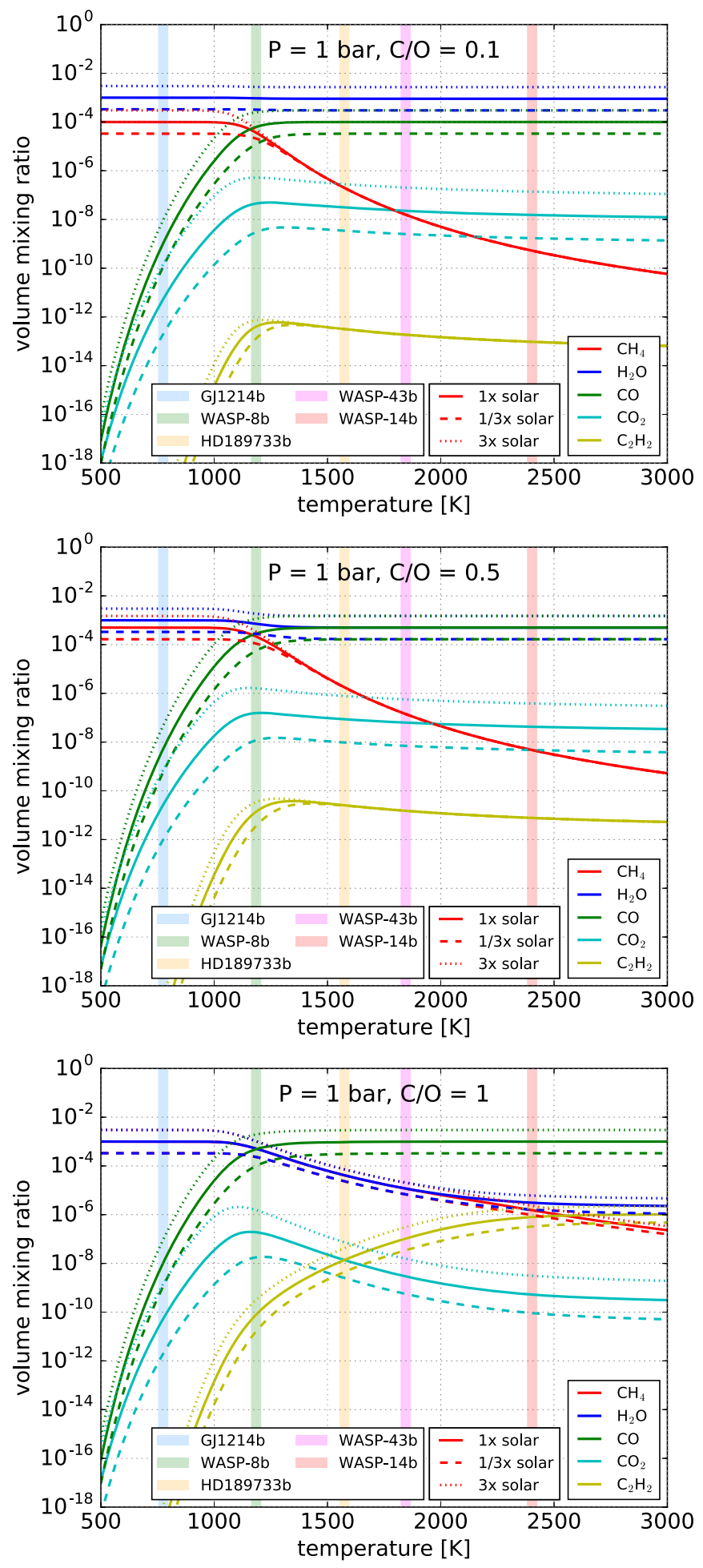

Figure 5. Elucidating the temperature dependence of the volume mixing ratios of the molecules used in the current study. For illustration, we set $P=1 \mathrm{bar}$ and explore $\mathrm{C} / \mathrm{O}=0.1$ (top panel), $\mathrm{C} / \mathrm{O}=0.5$ (middle panel), and $\mathrm{C} / \mathrm{O}=1$ (bottom panel). Within each panel, we explore the effects of varying the metallicity by $1 / 3 \times$ and $3 \times$ the solar value.

model atmosphere is being heated, which ultimately affects the temperature-pressure profile and synthetic spectrum. As both the PHOENIX and Kurucz stellar models do not extend across the entire wavelength range included in our calculations $(0.33 \mu \mathrm{m}-10 \mathrm{~cm})$, we patch them using a Planck function.

\subsection{Numerical Implementation}

The computationally intensive parts of HELIOS are written in CUDA C++ (Nickolls et al. 2008), a proprietary language extension of $\mathrm{C}++$ for general-purpose computations on suitable NVIDIA GPUs. The GPUs' main purpose is to provide a fast 2D graphical image where each pixel needs to be updated simultaneously, so their architecture is designed to maximize the throughput of parallel calculations and memory bandwidth. A radiative transfer problem is naturally amenable to parallelization because the flux propagation through the atmosphere can be computed for each wavelength separately if we assume coherent scattering (i.e., no change in the wavelength of the radiation). We also parallelize the interpolation of the precomputed $k$-distribution tables to determine the correct layer values. For further speed-up, the code offers the possibility to tabulate the Planck and the transmission functions at the model's wavelength values and a grid in $T(\Delta T=10 \mathrm{~K})$ and opacity $\left(\Delta \log _{10} \kappa=0.1\right)$, respectively. These grid resolutions are found to be sufficient for a converged behavior of the model (not shown here).

With this implementation of the temperature iteration, the procedure needs typically the following time: with an NVIDIA GeForce $750 \mathrm{M}$, the atmospheric temperatures converge within 2-15 minutes; with an NVIDIA K20 GPU, this takes between 0.5 and 4 minutes. These times have been found for a typical atmospheric setup with 101 layers and 300 wavelength bins, including a separate iteration for scattering during each numerical timestepping. Without scattering, the convergence times are usually a factor of 2 smaller. Once the converged temperature-pressure profile is found, the calculation of the emission spectrum with very high spectral resolution (3000 wavelength bins), as a post-processing step, takes less than 30 seconds. In our experience, we have found it to be sufficient to run HELIOS on a personal computer with an NVIDIA GPU (i.e., a GPU cluster is unnecessary).

\section{RESULTS}

We first subject HELIOS to a battery of tests. We then use it to address several lingering issues in the literature concerning the radiative transfer of exoplanetary atmospheres. Finally, we present $1 \mathrm{D}$, benchmark calculations for the emission spectra of six hot Jupiters (HD 189733b, WASP-8b, WASP-12b, WASP14b, WASP-33b, and WASP-43b) that serve as "null hypothesis" models.

By default, we use 300 wavelength bins and 101 nonisothermal layers in our calculations to solve for radiative equilibrium. The emission spectra are computed using 3000 bins and isothermal layers as a post-processing step. These bins are evenly distributed in wavenumber and cover a range of $0.1 \leqslant \lambda^{-1} \leqslant 30,000 \mathrm{~cm}^{-1}$, which corresponds to $0.33 \mu \mathrm{m}$ $\leqslant \lambda \leqslant 10 \mathrm{~cm}$. The layer pressures at the TOA and BOA are set at $1 \mu \mathrm{bar}$ and $1 \mathrm{kbar}$, respectively. Stellar heating is represented by a Planck function. The diffusivity factor is set to $\mathcal{D}=2$ and the redistribution efficiency factor is set to $f=2 / 3$. Isotropic scattering $\left(\omega_{0} \neq 0, g_{0}=0\right)$ and equilibrium chemistry with solar abundances are assumed. Unless otherwise stated, our fiducial model adopts these default parameter values.

\subsection{Tests}

To check HELIOS for consistency of the implementation, we focus on the case study of the super-Earth GJ 1214b. The parameter values used are listed in Table 2. 

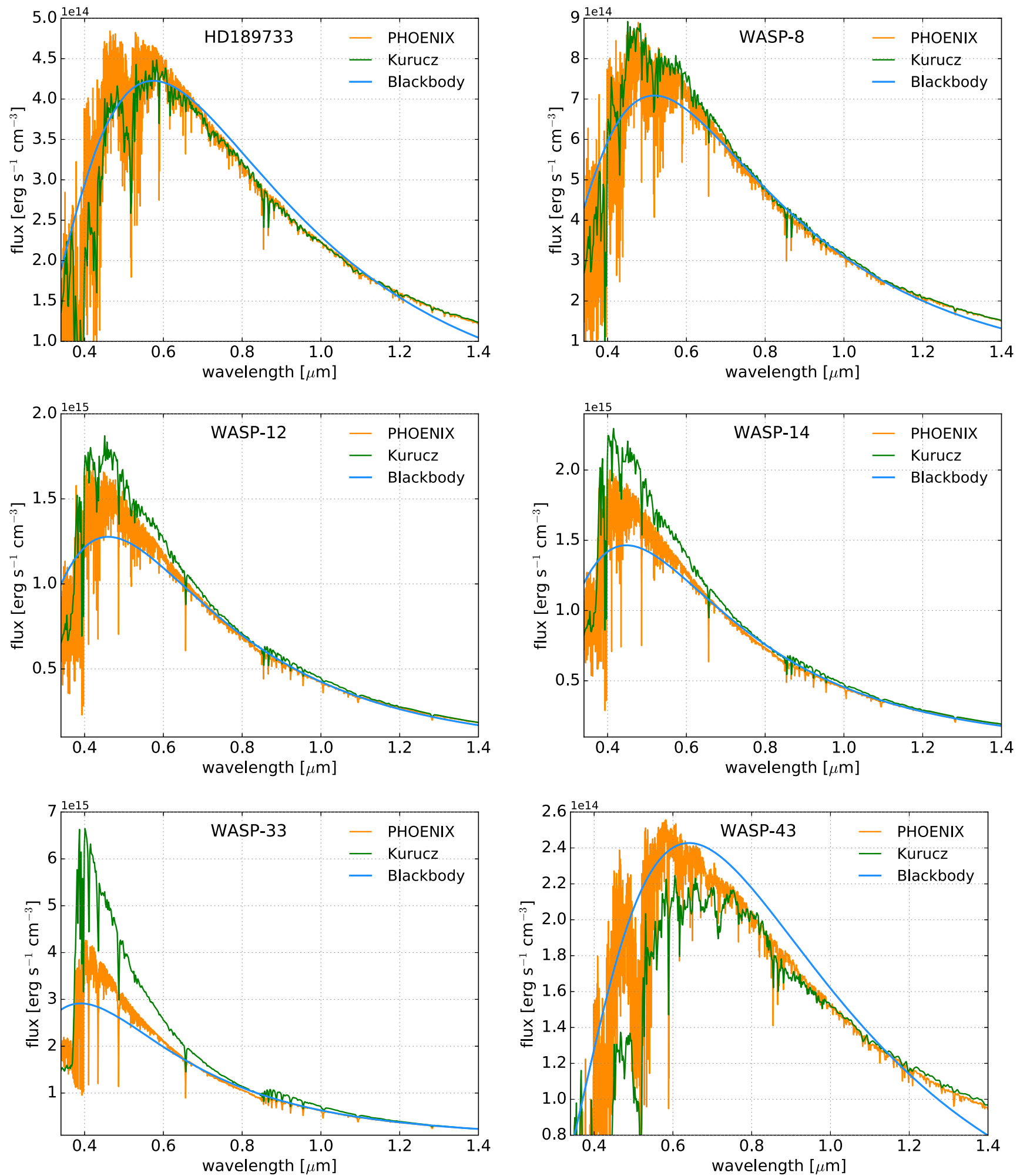

Figure 6. Comparison of the PHOENIX and Kurucz stellar models with the stellar blackbody function for the six hot Jupiters examined in the current study. Each stellar model was customized according to the specified stellar effective temperature, surface gravity, and metallicity, as stated in Table 2.

\subsubsection{Comparison to GJ 1214b Model of Miller-Ricci \& Fortney}

We test HELIOS against the results of Miller-Ricci \& Fortney (2010) for the planet GJ 1214b; they used the code originally developed by McKay et al. (1989) and Marley \& McKay (1999) for the atmospheres of solar system planets. It was later adapted to exoplanetary atmospheres by Fortney et al. (2005). They utilize a radiative transfer technique based on Toon et al. (1989), which is a multi-stream approach with a simplified two-stream solution for the scattering, further explained in Cahoy et al. (2010), and add a convection model for unstable atmospheric layers. Furthermore, Miller-Ricci \& 
Table 2

Planetary and Stellar Parameters used in this Study

\begin{tabular}{|c|c|c|c|c|c|c|c|}
\hline Object & GJ $1214 b^{a}$ & HD $189733 b^{b}$ & WASP- $8 b^{c}$ & WASP- $12 b^{d}$ & WASP- $14 b^{\mathrm{e}}$ & WASP- $33 b^{\text {f }}$ & WASP-43b \\
\hline Mean molecular mass $\bar{m}\left(m_{\mathrm{p}}\right)$ & \multicolumn{7}{|c|}{$2.4^{\mathrm{h}}$} \\
\hline Surface gravity $g\left(\mathrm{~cm} \mathrm{~s}^{-2}\right)$ & 768 & 1950 & $5510^{\mathrm{i}}$ & 1164 & 10233 & 2884 & 4699 \\
\hline Orbital separation $a(\mathrm{au})$ & 0.01411 & 0.03142 & 0.0801 & 0.02293 & 0.036 & 0.0259 & 0.0152 \\
\hline Effective temp. $T_{\text {eff }}^{\mathrm{j}}(\mathrm{K})$ & $775\left(660^{\mathrm{k}}\right)$ & 1575 & 1185 & 3241 & 2403 & 3494 & 1845 \\
\hline Planet. radius $R_{\mathrm{pl}}\left(R_{\mathrm{Jup}}\right)$ & 0.2479 & 1.216 & 1.038 & 1.776 & 1.281 & 1.679 & 1.036 \\
\hline Stellar temp. $T_{\star}(\mathrm{K})$ & $3252\left(3026^{\mathrm{k}}\right)$ & 5050 & 5600 & 6300 & 6475 & 7430 & 4520 \\
\hline Stellar radius $R_{\star}\left(R_{\odot}\right)$ & 0.211 & 0.805 & 0.945 & 1.595 & 1.306 & 1.509 & 0.667 \\
\hline Stellar surface gravity $\log g_{\star}\left(\mathrm{cm} \mathrm{s}^{-2}\right)$ & 5.04 & 4.53 & 4.5 & 4.16 & 4.07 & 4.3 & 4.645 \\
\hline Stellar metallicity $[\mathrm{F} / \mathrm{H}]$ & 0.13 & 0.0 & 0.2 & 0.2 & 0.0 & 0.0 & 0.0 \\
\hline
\end{tabular}

Notes.

${ }^{\text {a }}$ Bouchy et al. (2005), Anglada-Escudé et al. (2013), Harpsøe et al. (2013).

${ }^{\mathrm{b}}$ Southworth (2010), de Kok et al. (2013), Boyajian et al. (2015).

${ }^{\mathrm{c}}$ Queloz et al. (2010).

${ }^{\mathrm{d}}$ Hebb et al. (2009), Chan et al. (2011).

e Joshi et al. (2009).

${ }^{\mathrm{f}}$ Collier Cameron et al. (2010), Kovács et al. (2013), Lehmann et al. (2015).

${ }^{\mathrm{g}}$ Gillon et al. (2012).

${ }^{\mathrm{h}}$ Our choice value for a hydrogen-dominated atmosphere.

i This value has been obtained from Newton's law of gravity assuming a spherical shape of the planet and neglecting rotation.

${ }^{\mathrm{j}}$ Assuming dayside heat redistribution using a factor $f=2 / 3$.

${ }^{\mathrm{k}}$ This value is used for the model comparison with Miller-Ricci \& Fortney (2010).

Fortney (2010) use the opacities associated with $\mathrm{H}_{2} \mathrm{O}, \mathrm{CO}_{2}$, $\mathrm{CO}, \mathrm{CH}_{4}$, and $\mathrm{NH}_{3}$ (Freedman et al. 2008), as well as the CIA opacities associated with $\mathrm{H}_{2}-\mathrm{H}_{2}, \mathrm{H}_{2}-\mathrm{He}, \mathrm{H}_{2}-\mathrm{CH}_{4}$, and $\mathrm{CO}_{2}-$ $\mathrm{CO}_{2}$. Their chemistry model is taken from Lodders \& Fegley (2002, 2006) and they include a treatment of Rayleigh scattering by molecular hydrogen. Still, we choose to compare HELIOS with the results of Miller-Ricci \& Fortney (2010), because the employed radiative transfer technique and also the list of absorbers, together with the Rayleigh scattering, are similar to ours.

As a reference, we take their solar-abundance model that has a dayside-averaged temperature of $660 \mathrm{~K}$ (see the red, dashed curve in their Figure 1). To permit any reasonable comparison, we use the same astronomical parameters as Miller-Ricci \& Fortney (2010). For instance, we set the stellar temperature to $3026 \mathrm{~K}$ and tune the redistribution parameter $f$ (in this test only) so that the dayside-effective temperature attains $660 \mathrm{~K}$ as in their setup. Furthermore, to mimic their use of a stellar spectrum for GJ 1214 from Hauschildt et al. (1999) we also employ a PHOENIX stellar spectrum (from the updated online database) for the same stellar parameters, extrapolated by a blackbody fit to cover the whole wavelength range.

In Figure 7, left panel, we show the temperature-pressure profiles for GJ 1214b by Miller-Ricci \& Fortney (2010) and as computed with HELIOS. There is excellent agreement around $P=10^{-2}-1$ bar-essentially, the calculations produce infrared photospheric temperatures that coincide. At $P>1$ bar, the HELIOS temperature-pressure profile is about $200 \mathrm{~K}$ hotter. We suspect that this discrepancy is due to our simpler treatment of the opacities, as we consider only four molecules. This leads to greater transparency, particularly in the visible wavelengths of our model atmosphere, which in turn produces more heating in the deep atmosphere. To support this hypothesis, we have successfully reproduced the deep atmospheric structure of Miller-Ricci \& Fortney (2010) by artificially introducing an opacity of $6 \times 10^{-4} \mathrm{~cm}^{2} \mathrm{~g}^{-1}$ to the shortwave below $1 \mu \mathrm{m}$ (see green dashed curve in Figure 7). Since our model does not have any convective treatment, we cannot reproduce the adiabat in their model at the bottom boundary. However, by introducing an internal heat flux, we can somewhat mimic their deep temperatures (shown for $T_{\text {intern }}=60 \mathrm{~K}$ ).

In the right panel of Figure 7 we show the ratio of the planetary and the stellar emission for the model of Miller-Ricci \& Fortney (2010) and for ours. The spectra are of the same magnitude and show similar trends. Their results show a larger variation in intensity across wavelength, particularly enabling emission from deeper, and thus hotter, atmospheric regions. This could be a consequence of several factors: differences in employed molecular line lists, combination of the opacities, chemistry model, or the stellar spectrum. Considering all those components, it is not surprising that the individual spectral features do not match perfectly, and we conclude that HELIOS is still rather consistent with the results of Miller-Ricci \& Fortney (2010). For completion, we show both the spectrum of our fiducial setup and the one with an added artificial shortwave opacity. As expected, those are very similar because the models only differ slightly around the emitting photosphere.

\subsubsection{Trends Associated with Scattering}

As a further consistency check of HELIOS, we examine calculations with idealized descriptions of scattering and check whether the trends match our physical intuition.

For illustration, we set $\omega_{0}=0.5$ across all wavelengths. We then examine models with $g_{0}=-0.5,0$, and 0.5 , which are also constant across all wavelengths. We emphasize that the two-stream solutions used in HELIOS, which are taken from Heng et al. (2014), are generally able to take $\omega_{0}$ and $g_{0}$ as input functions (rather than just scalars/numbers).

Figure 8 shows the fiducial pure absorption model compared against the three models with idealized descriptions of isotropic, backward, and forward scattering. For $g_{0}=-0.5$ 

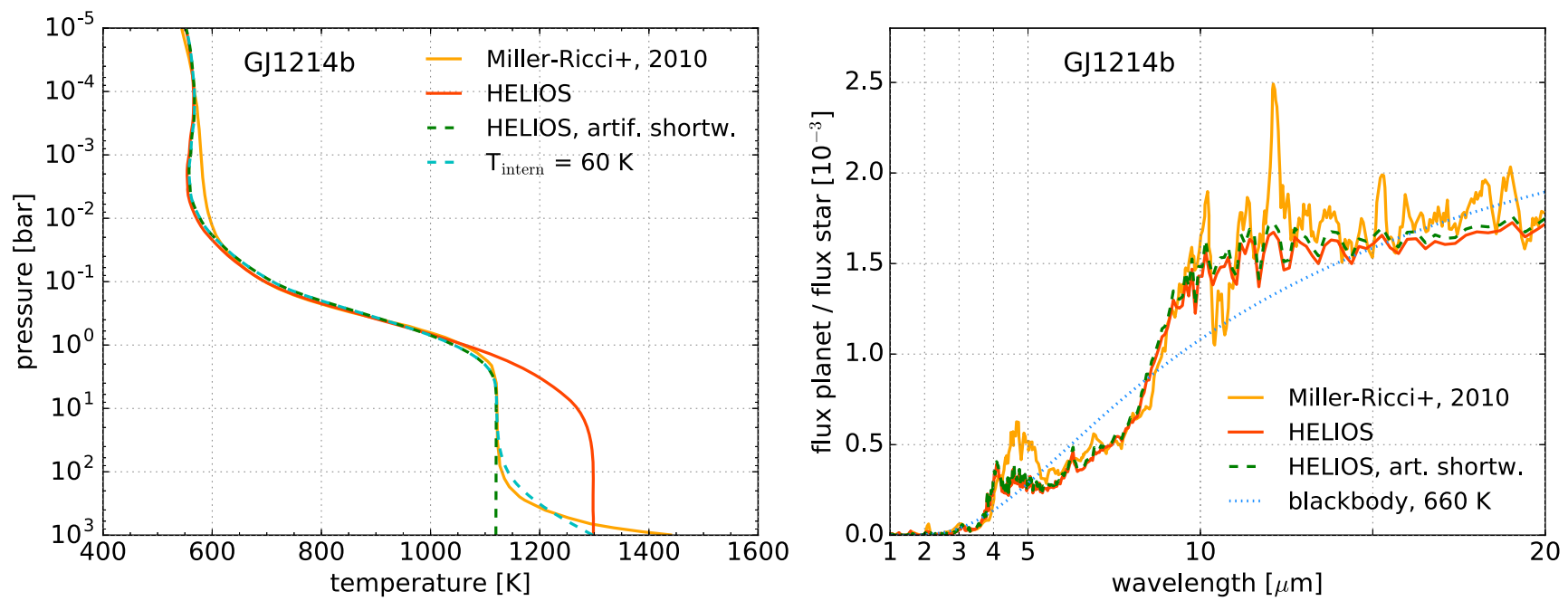

Figure 7. Comparison with the atmospheric model of GJ 1214b from Miller-Ricci \& Fortney (2010). The left panel shows the dayside temperature-pressure profile at $T_{\text {eff }}=660 \mathrm{~K}$. The temperatures in the infrared photosphere $\left(\sim 10^{-2}-1\right.$ bar) match very well. We also reproduce the temperatures in the deep atmosphere when an artificial opacity of $6 \times 10^{-4} \mathrm{~cm}^{2} \mathrm{~g}^{-1}$ is inserted into the visible wavelengths (dashed curve). We can mimic the convective tail by adding internal heat flux, shown here for $T_{\text {intern }}=60 \mathrm{~K}$ (cyan). The right panel depicts the corresponding planetary emission for three models of the left panel, together with a blackbody emission at the same effective temperature for comparison. The spectrum of Miller-Ricci \& Fortney (2010) shows more pronounced features, but overall has the same magnitude. The HELIOS runs are similar because the main temperature difference lies below the emitting photosphere.
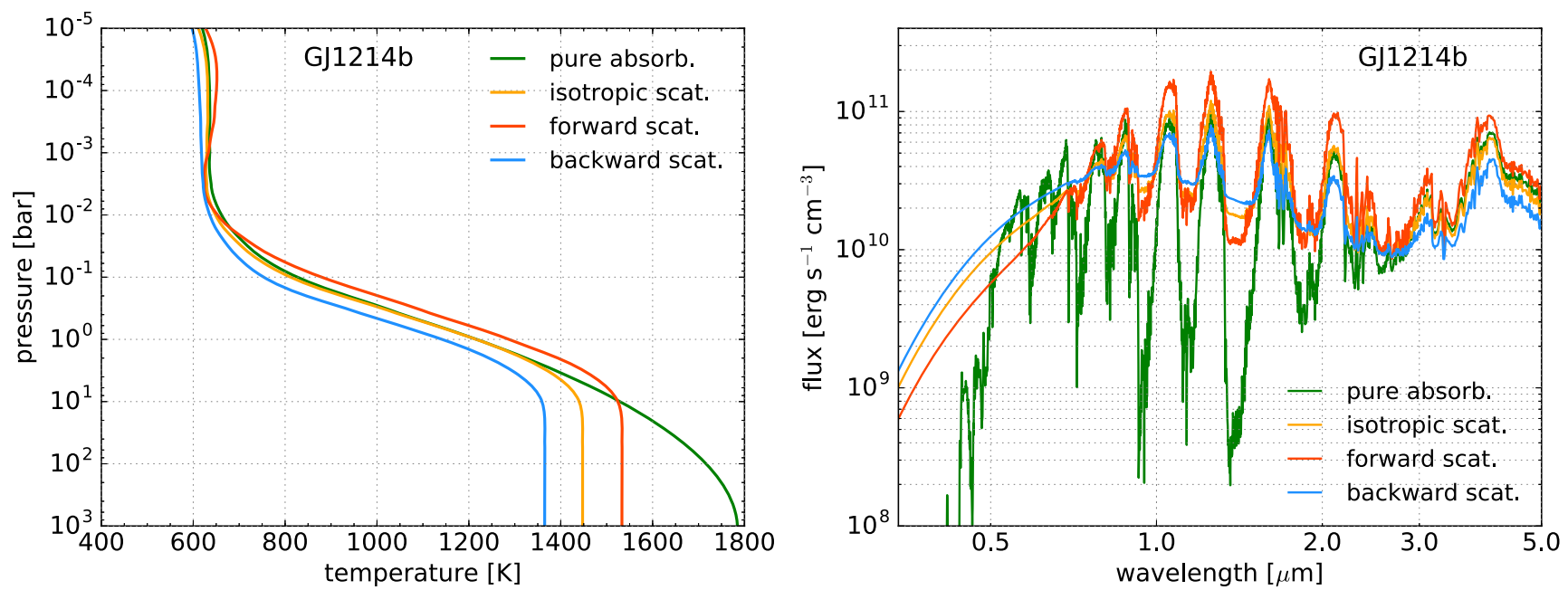

Figure 8. Consistency check of HELIOS by examining the temperature-pressure profiles (left panel) and synthetic spectra (right panel) in the idealized limits of scattering: $g_{0}=0$ (isotropic scattering), $g_{0}=0.5$ (forward scattering), and $g_{0}=-0.5$ (backward scattering). For illustration, we set $\omega_{0}=0.5$. Both the singlescattering albedo $\left(\omega_{0}\right)$ and scattering asymmetry factor $\left(g_{0}\right)$ are assumed to be constant across wavelength, but we note that the two-stream solutions we implemented allow for them to generally be specified as functions of wavelength, temperature, and pressure. The qualitative trends associated with the temperature-pressure profiles and synthetic spectra are consistent with physical expectations (see text).

and 0 , scattering generally shifts the absorption profile of starlight upward (toward lower pressures), which cools the model atmosphere. As the scattering shifts from being isotropic to being backward, the deep atmosphere becomes cooler. We also observe a trend of the reflected light at $\lesssim 1 \mu \mathrm{m}$ being the strongest for backward scattering, but of the thermal emission at $\gtrsim 1 \mu \mathrm{m}$ being the strongest for forward scattering, which is expected.

Scattering also has the general effect of muting the spectral features in the synthetic spectra. It effectively raises the level of the infrared continuum. This effect is stronger as the scattering becomes more backward-dominated (Figure 8). Such an effect mimics the presence of aerosols or condensates. Overall, these expected trends provide a "proof-of-concept" validation of HELIOS.

\subsubsection{Isothermal versus Nonisothermal Layers}

An essential ingredient of 1D models of atmospheres in radiative equilibrium is the number of layers used in the computation. We perform a series of convergence tests by considering different numbers of layers and employing models with isothermal versus nonisothermal layers. We again use the parameters of GJ 1214b as an illustration.

Figure 9 shows the temperature-pressure profiles associated with models having 51, 201, and 1001 isothermal layers, and also those with 21,101 , and 501 nonisothermal layers. First, we note that the temperature-pressure profiles of the models with nonisothermal layers coincide (with differences of less than $3 \mathrm{~K}$ ), implying that 21 nonisothermal layers are sufficient to attain convergence. By contrast, even with 1001 layers, no convergence is seen for the models with isothermal layers. 

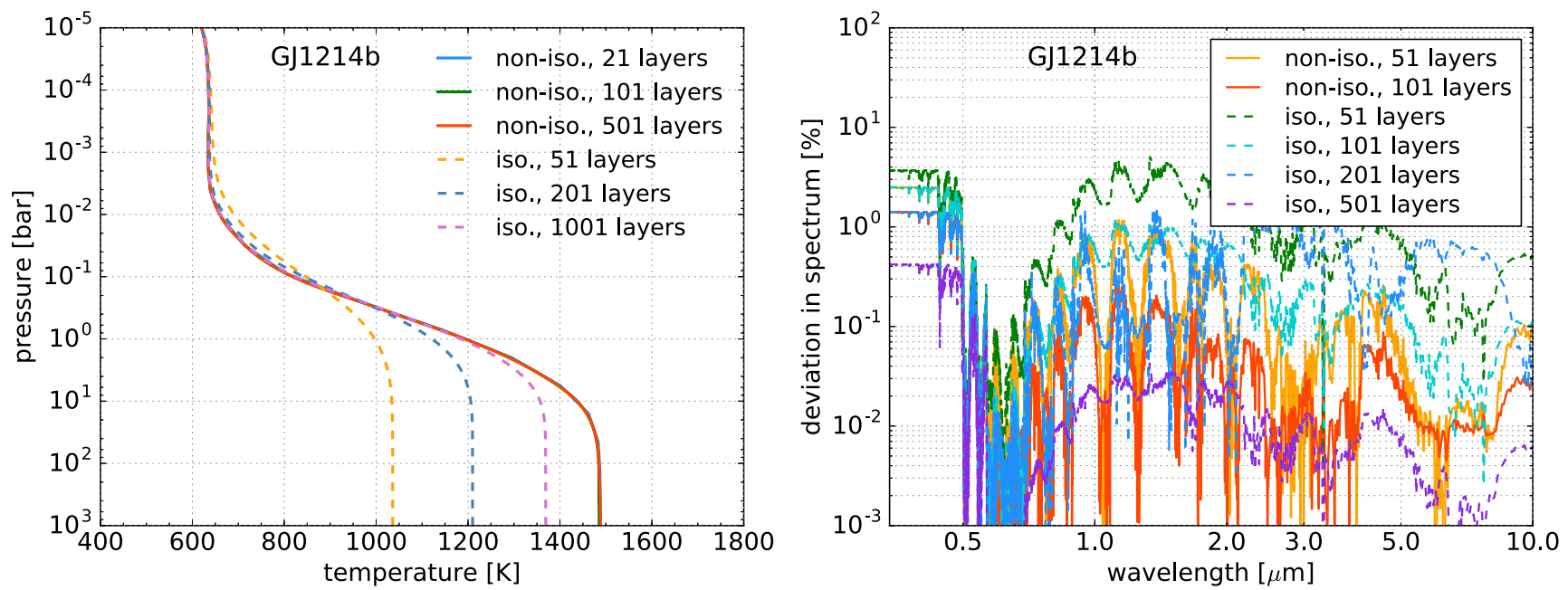

Figure 9. Resolution tests to determine the minimum number of isothermal vs. nonisothermal layers needed for numerical convergence. The left panel shows various temperature-pressure profiles computed using 51, 201, and 1001 isothermal layers vs. 21, 101, and 501 nonisothermal layers, demonstrating that the use of isothermal layers is not an efficient approach. The right panel shows the deviation or error in the synthetic spectrum, as a function of wavelength, using the model with 501 nonisothermal layers as a reference.

These results illustrate the superiority of using nonisothermal layers. We recover the same behavior even when a different case study (e.g., WASP-12b) is considered (not shown).

Next, we compute the synthetic spectrum of the model with 501 nonisothermal layers and use it as a reference. We then consider models with 51 and 101 nonisothermal layers, as well as models with 51, 101, 201, and 501 isothermal layers. For each model, we compute the deviation in the synthetic spectrum, from the reference model, as a function of wavelength. Figure 9 shows that, as expected, the deviation decreases as the resolution increases. Only the model with 51 isothermal layers produces deviations that exceed $1 \%$ in the flux. The model with 101 isothermal layers produces deviations that are typically less than $1 \%$. Since models with isothermal layers are faster to compute, this motivates us to adopt a model with 101 isothermal layers for our post-processing step. In other words, we use nonisothermal layers to iterate for radiative equilibrium. Upon attaining radiative equilibrium, we postprocess the converged temperature-pressure profile, using a model with 101 isothermal layers, to produce synthetic spectra.

\subsubsection{Obtaining Convergence for the $k$-distribution Tables}

Another essential ingredient of 1D models of atmospheres in radiative equilibrium is the spectral resolution used in constructing the opacity function, which is then used to construct the $k$-distribution tables. We wish to investigate the errors associated with using different spectral resolutions. The reference case is taken to be a model with a spectral resolution of $10^{-5} \mathrm{~cm}^{-1}$. We examine models with resolutions of $10^{-1}$, $10^{-2}, 10^{-3}$, and $10^{-4} \mathrm{~cm}^{-1}$ and compare the errors in the synthetic spectra, after we have iterated for radiative equilibrium, as a function of wavelength, relative to the reference. As we are using 3000 wavelength bins, these sampling resolutions correspond to $10^{2}, 10^{3}, 10^{4}$, and $10^{5}$ points per bin, respectively.

Figure 10 shows our results for the case studies of GJ $1214 \mathrm{~b}$ and WASP-12b, which were illustrated to span the range of temperatures for the currently characterizable exoplanetary atmospheres. We find the expected trend that the error decreases as the resolution increases from $10^{-1}$ to $10^{-4} \mathrm{~cm}^{-1}$.
Using a spectral resolution of only $10^{-1} \mathrm{~cm}^{-1}\left(10^{-2} \mathrm{~cm}^{-1}\right)$ results in errors that are $>10 \%(\sim 1 \%-10 \%)$ in the near-infrared flux. To reduce the error to $\sim 1 \%$, we find a minimum resolution of $10^{-3} \mathrm{~cm}^{-1}$ to be required in our model. This value might change if one is using opacity sampling. We also show the error in the spectra produced by purely post-processing the temperature profile of the reference case, which demonstrates that the errors are not merely associated with iterating for radiative equilibrium.

\subsubsection{Using the Correct Value of the Diffusivity Factor}

As discussed previously, one may obtain an exact solution of the radiative transfer equation, without invoking the two-stream approximation, only in the limit of pure absorption. This solution may be compared to two-stream calculations with different assumed values of the diffusivity factor.

Amundsen et al. (2014) have recently advocated the use of $\mathcal{D}=1.66$ from comparison of their two-stream calculations to a different set of calculations computed using the discreteordinates radiative transfer method. Armstrong (1969) also advocated $\mathcal{D}=1.66$ based on radiative transfer calculations of water in the atmosphere of Earth. However, the correct value for $\mathcal{D}$ should depend on the vertical resolution of the model (see Figure 1), which motivates us to perform our own comparisons.

Figure 11 displays the computed temperature-pressure profiles and the error in the resulting synthetic spectrum for GJ $1214 \mathrm{~b}$ for $\mathcal{D}=1.66,1.8,1.9$, and 2 compared to the exact solution. Regarding temperature, the $\mathcal{D}=1.9$ and 2 models produce the best match to the exact solution. However, $\mathcal{D}=2$ leads, on average, to the smallest error in the spectrum. We also consider the same set of calculations for a hotter exoplanet, WASP-12b. In this case, $\mathcal{D}=2$ clearly produces the best match to the exact solution in terms of the temperature as well as the spectrum. In general, the error in the spectrum is smaller than for the cooler planet. It is not surprising that $\mathcal{D}=2$ provides the most accurate results, because we expect the diffusivity factor to approach a value of 2 when the vertical resolution of the model is sufficient (see Figure 1), i.e., the 

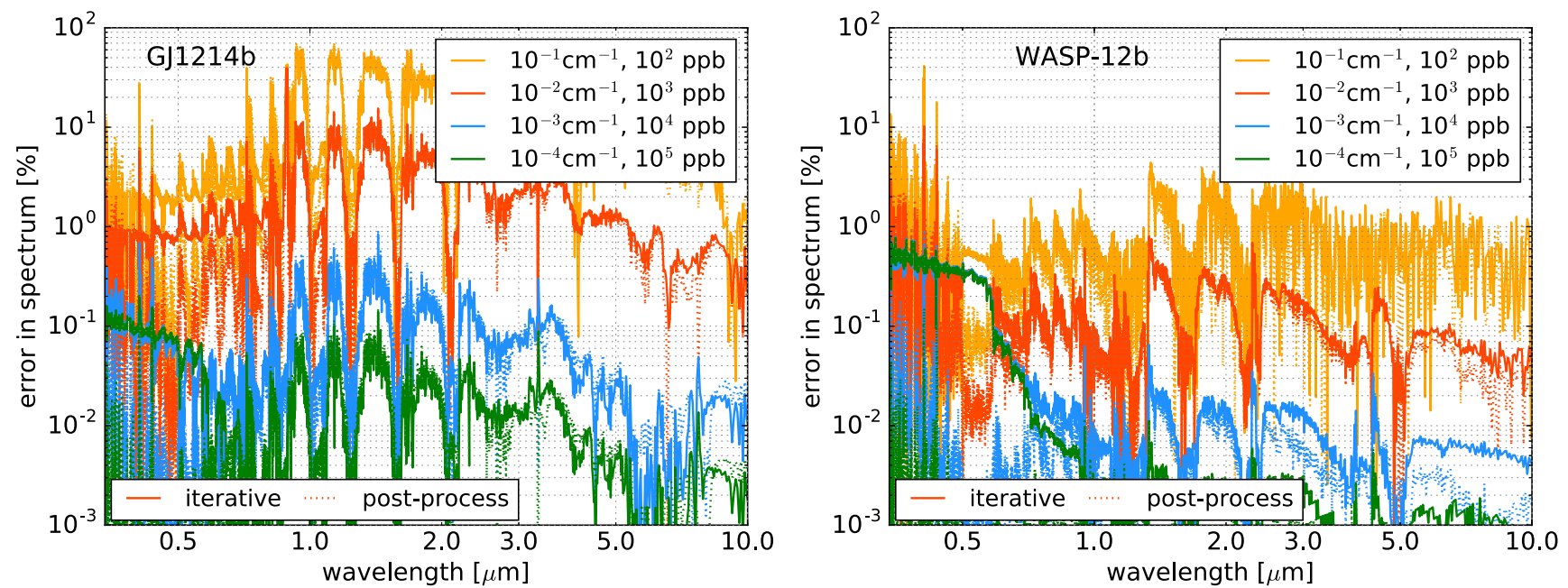

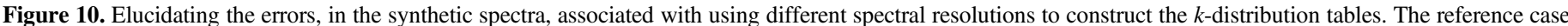

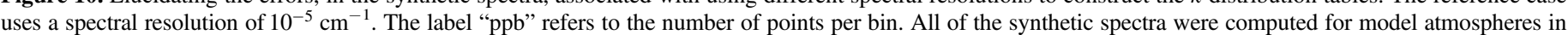

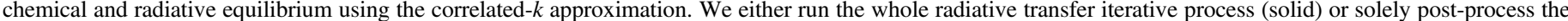

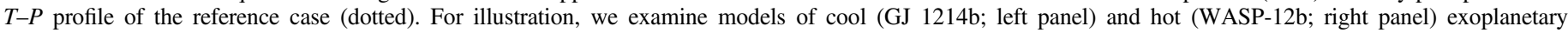
atmospheres.

difference in optical depth between the layers is small, at least in the photospheric regions of the atmosphere.

\subsection{Testing the Null Hypothesis and Variations on a Theme: Benchmark 1D Models for Hot Jupiters}

Despite heroic efforts to obtain data for exoplanetary atmospheres, exoplanets are spatially unresolved point sources-and will probably remain so for the foreseeable future-although phase curves and eclipse maps provide some spatial information. As a first approach, theorists have resorted to interpreting the spectra of exoplanetary atmospheres using simple models: 1D, plane-parallel, just as we have constructed. There is a precedent of using 1D models to interpret spectra (see Introduction for references). As a null hypothesis, we make the following assumptions: chemical equilibrium, radiative equilibrium (which we solve for using HELIOS), and solar abundances. This would be the second simplest model after a blackbody emission spectrum (Hansen et al. 2014). Upon constructing the null hypothesis, we then examine variations in the metallicity and $\mathrm{C} / \mathrm{O}$.

We have chosen the sample of hot Jupiters to include in this analysis based on a literature search for planets with nonblackbody emission spectra. We have started from Hansen et al. (2014), which catalogs all planets with secondary eclipse measurements in at least two bandpasses as of 2014. They found seven planets that are poorly fit by a blackbody model. We also searched for any more recent nonblackbody results. To select the most precise, reliable measurements from our search, we consider space-based data only. We have also stipulated that the data were reduced with state-of-the-art techniques. Specifically, we only consider Spitzer results that used sophisticated models of the intrapixel sensitivity such as BLISS mapping or pixel-level decorrelation (Stevenson et al. 2012; Deming et al. 2015). This approach has been demonstrated to be the best practice in Spitzer data analysis (Ingalls et al. 2016). This search has resulted in the selection of six planets: HD 189733b, WASP-8b, WASP-12b, WASP-14b, WASP-33b, and WASP-43b. Their model parameter values and spectral data sources are shown in Tables 2 and 3, respectively.

Figure 12 shows the null-hypothesis models for all six studies. We have computed synthetic spectra and temperaturepressure profiles using a stellar blackbody, a Kurucz stellar model, and a PHOENIX stellar model. All of the stellar models were customized for each case study by specifying, as input parameters, the stellar effective temperature, surface gravity, and metallicity. The synthetic spectra in all three cases are qualitatively similar. The largest difference occurs between 3 and $10 \mu \mathrm{m}$. These differences appear to be more pronounced for the hottest hot Jupiters (i.e., WASP-12b and WASP-33b). Interestingly, the choice of stellar model affects the strength of the water-band features between 1.5 and $2.5 \mu \mathrm{m}$, which are partially probed by the WFC3 instrument on the Hubble Space Telescope. This discrepancy between the models is somewhat apparent for HD 189733b and WASP-43b. The shapes of the temperature-pressure profiles, in all six cases, are very similar with the largest discrepancies in either the very high, optically thin layers or deep, optically thick layers, which are less important for the planetary emission.

Overall, HD 189733b appears to be consistent with a null hypothesis and its dayside emission spectrum is reasonably described by a 1D, plane-parallel model in chemical and radiative equilibrium with solar metallicity. WASP-43b is fairly well described by the null hypothesis. However, our models for WASP-8b, WASP-12b, WASP-14b, and WASP-33b consistently underpredict the infrared fluxes. These discrepancies could be either due to an insufficient implementation of opacity (lacking partial molecular absorption, aerosol extinction, or inaccurate line profiles) or due to a limited methodological framework, lacking chemical disequilibrium (which requires a self-consistent calculation coupled to a chemical kinetics solver), radiative disequilibrium (which requires another selfconsistent calculation coupled to atmospheric dynamics), or non-1D effects (which a 1D model prescription with $f$ cannot characterize and which would ideally require coupling to a 3D spatially resolved, general circulation model). We will defer this investigation to future work. 

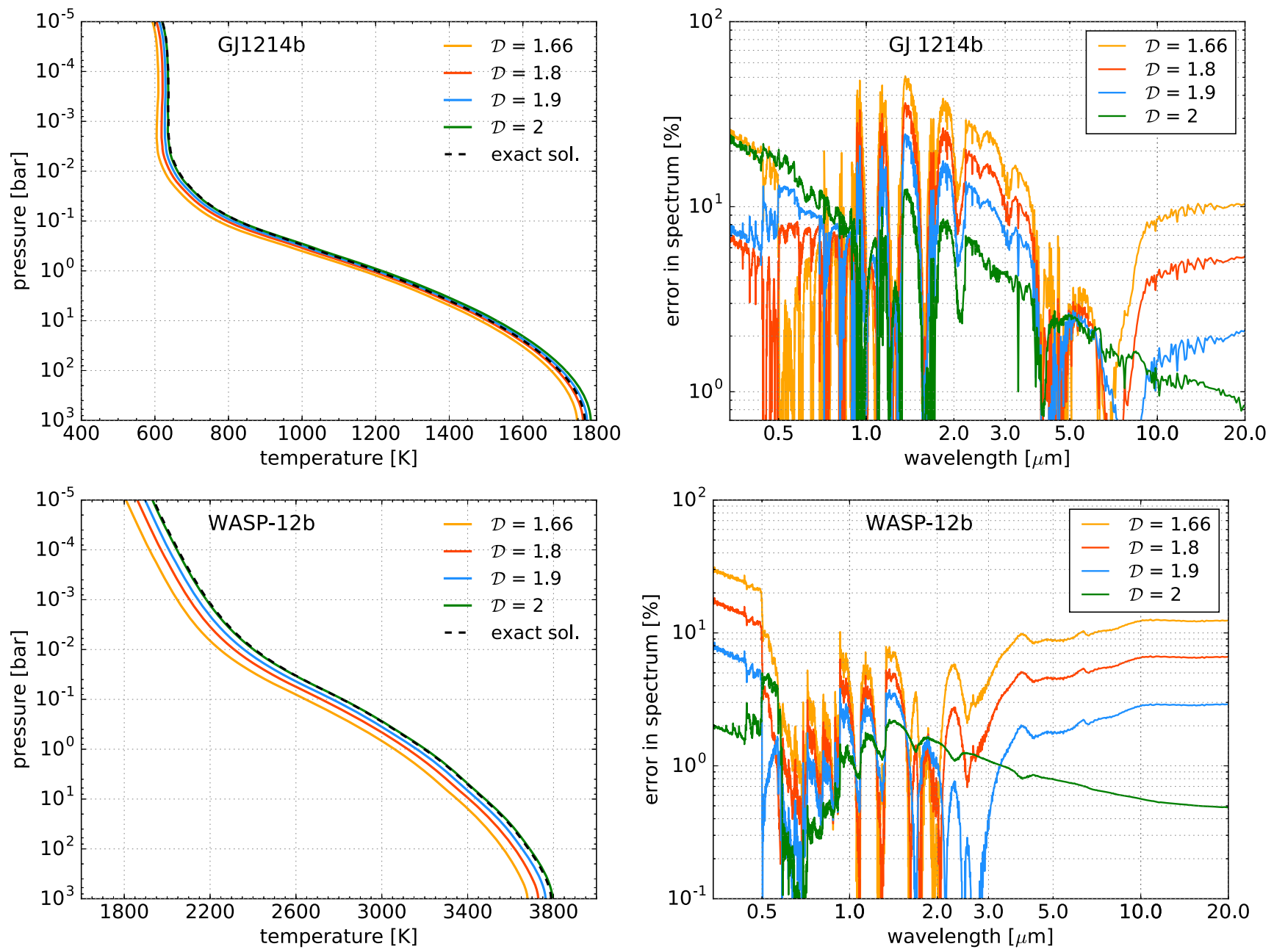

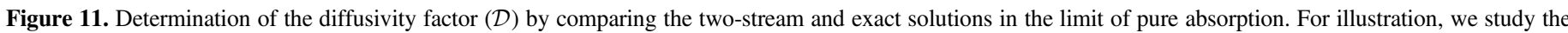

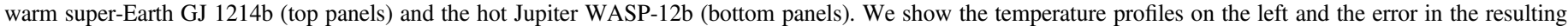

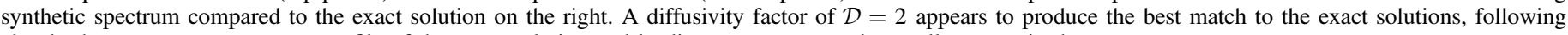
closely the temperature-pressure profile of the exact solution and leading on average to the smallest error in the spectrum.

Table 3

Spectral Data Sources

\begin{tabular}{ll}
\hline \hline Planet & Source \\
\hline HD 189733b & Crouzet et al. (2014), Todorov et al. (2014) \\
WASP-8b & Cubillos et al. (2013) \\
WASP-12b & Stevenson et al. (2014) \\
WASP-14b & Blecic et al. (2013) \\
WASP-33b & Deming et al. (2012), Haynes et al. (2015) \\
WASP-43b & Blecic et al. (2014), Kreidberg et al. (2014) \\
\hline
\end{tabular}

For further variations on the theme, we retain the PHOENIX stellar models because they offer higher spectral resolution and more updated atomic/molecular line lists than the Kurucz stellar models. In Figure 13, we repeat our calculations with $1 / 3 \times, 1 \times$, and $3 \times$ solar metallicity. We find the expected trend that a higher metallicity leads to generally hotter model atmospheres, which has the effect of strengthening the nearinfrared water-band features. However, compared to the null hypothesis, decreasing or increasing the metallicity by a factor of 3 appears to have a minimal effect on the synthetic spectra, which is consistent with the retrieval analysis conducted for WASP-43b in Kreidberg et al. (2014), where they obtain similar metallicity uncertainties based on data constraints. Our conclusions are qualitatively identical to those visible in Figure 12.

Varying the $\mathrm{C} / \mathrm{O}$ has a more marked effect, as we show in Figure 14. Specifically, we examine water-rich $(\mathrm{C} / \mathrm{O}=0.1)$, solar-abundance $(\mathrm{C} / \mathrm{O}=0.5)$, and $\mathrm{C} / \mathrm{O}=1$ scenarios. Generally, we find that the $\mathrm{C} / \mathrm{O}=1$ models have consistently colder temperature-pressure profiles, due to the lower abundance of $\mathrm{H}_{2} \mathrm{O}$ as the oxygen atom is preferentially sequestered by $\mathrm{CO}$, at high temperatures, compared to the water-rich and solar-abundance models. The increasing abundance of $\mathrm{CO}$ also leads to stronger absorption features at $2.3,4.5$, and $4.8 \mu \mathrm{m}$, which render the model atmospheres darker (i.e., they have less flux in these bands). This transition to the stronger $\mathrm{CO}$ features is more pronounced in the hotter objects (WASP-12b and WASP-33b). Our qualitative conclusions appear to be unchanged: our models for WASP-8b, WASP-12b, WASP$14 \mathrm{~b}$, and WASP-33b still underpredict the infrared fluxes. It is somewhat difficult to judge whether the data favor the waterrich or $\mathrm{C} / \mathrm{O}=1$ models, for HD $189733 \mathrm{~b}$ and WASP-43b, without running a detailed atmospheric retrieval model, which we again defer to future work. 

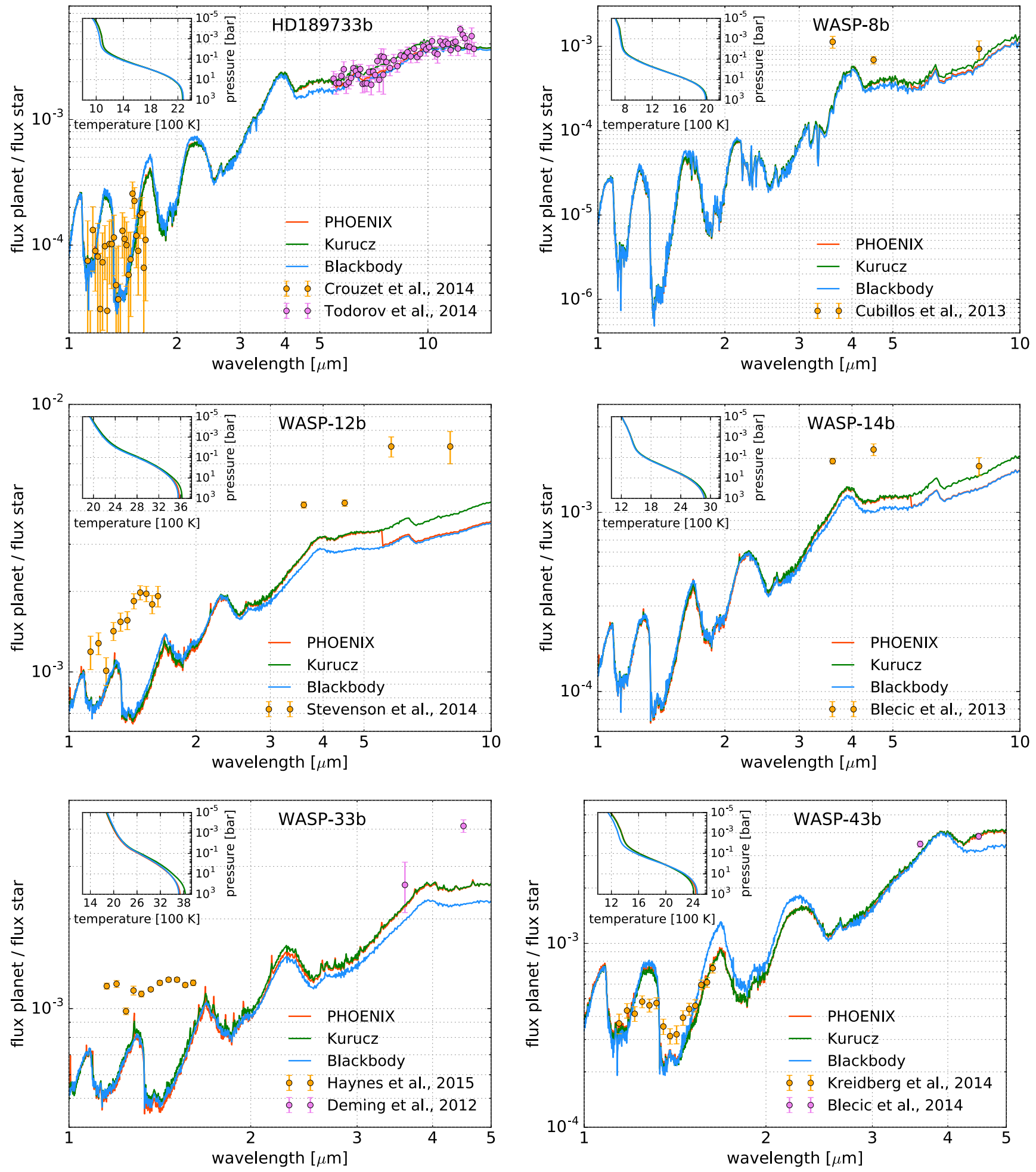

Figure 12. Null-hypothesis models for the six hot Jupiters in our current study: 1D, plane-parallel model atmospheres in chemical and radiative equilibrium, with solar metallicity/abundances. The predicted dayside emission spectra were compared to published data (see text for details). For each case study, we computed three models using the PHOENIX and Kurucz stellar models as well as a stellar blackbody. For each assumption of the stellar irradiation flux, we iterated the model atmosphere to attain radiative equilibrium (see text for details).

\section{SUMMARY, DISCUSSION, AND CONCLUSIONS}

\subsection{Summary}

We have presented the new, extensible code, HELIOS, which solves the equation of radiative transfer for a 1D, planeparallel atmosphere that allows for nonisotropic scattering via the specification of the functional forms of the single-scattering albedo and the scattering asymmetry factor. It uses a staggered spatial grid with the options of specifying isothermal or nonisothermal layers. We have used HELIOS-K (Grimm \& Heng 2015) to compute the opacities of the four molecules that are active in the infrared, and combined those by weighting 

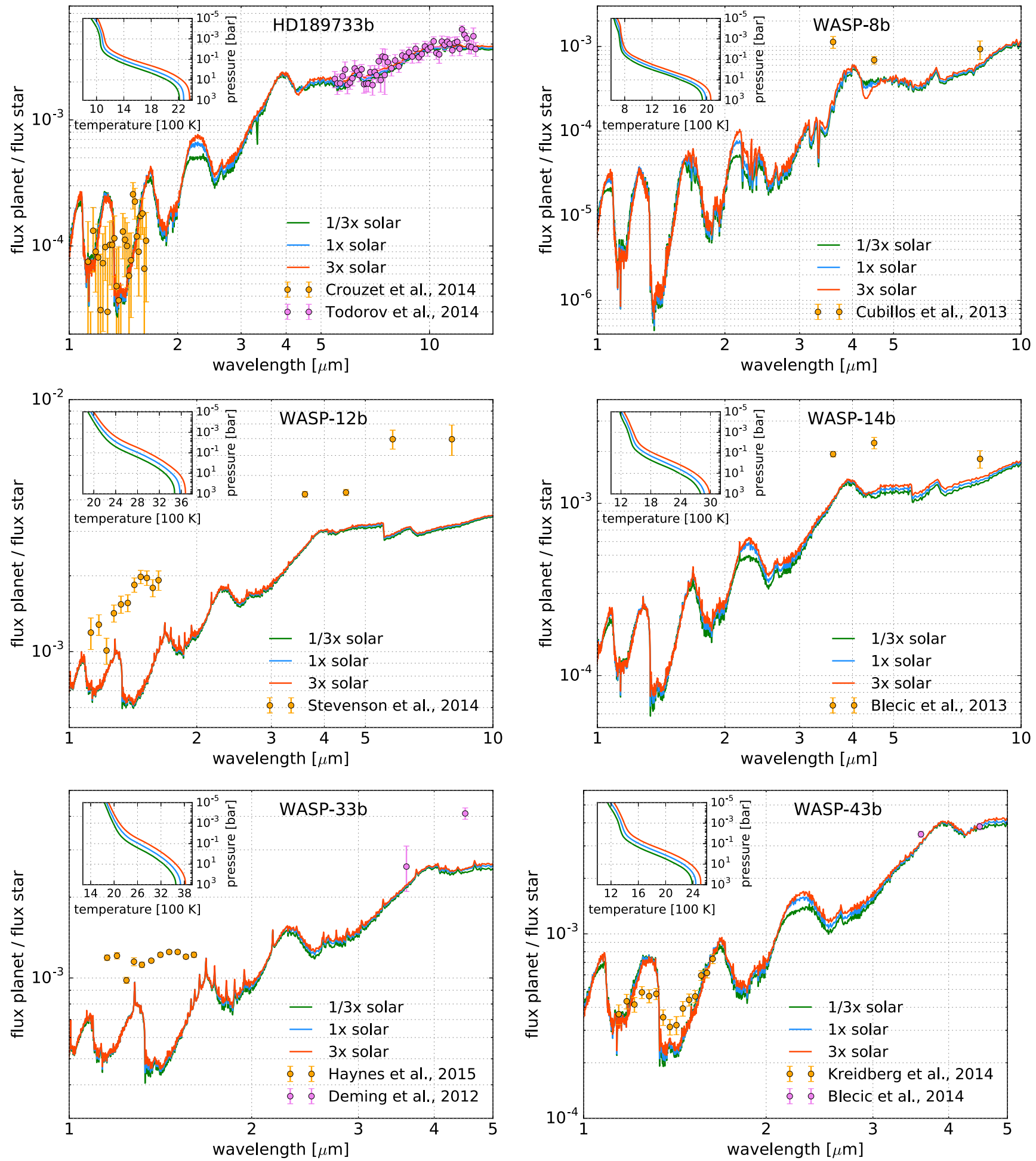

Figure 13. Same as Figure 12, but using only the PHOENIX stellar model and examining the effects of varying the metallicity of the model atmospheres.

them with the validated analytical formulae of Heng \& Lyons (2016) and Heng \& Tsai (2016) for equilibrium chemistry. In order to combine the various gaseous absorbers we have employed a correlated- $k$ approximation, which assumes perfect correlation between the molecular bands. The boundary conditions are the stellar irradiation flux at the top of the model atmosphere and the internal heat flux at the bottom. HELIOS further allows for the stellar irradiation flux to be specified as a simple Planck function or from a stellar model (e.g., Kurucz, PHOENIX). We have constructed and optimized HELIOS to run on GPUs, which allows for fast computation on a single machine. We have exploited this efficiency to explore the parameter space of stellar type, metallicity, and $\mathrm{C} / \mathrm{O}$ ratio.

\subsection{Comparison to Previous Work}

Several groups have made contributions to a rich body of literature on self-consistent radiative transfer models in 

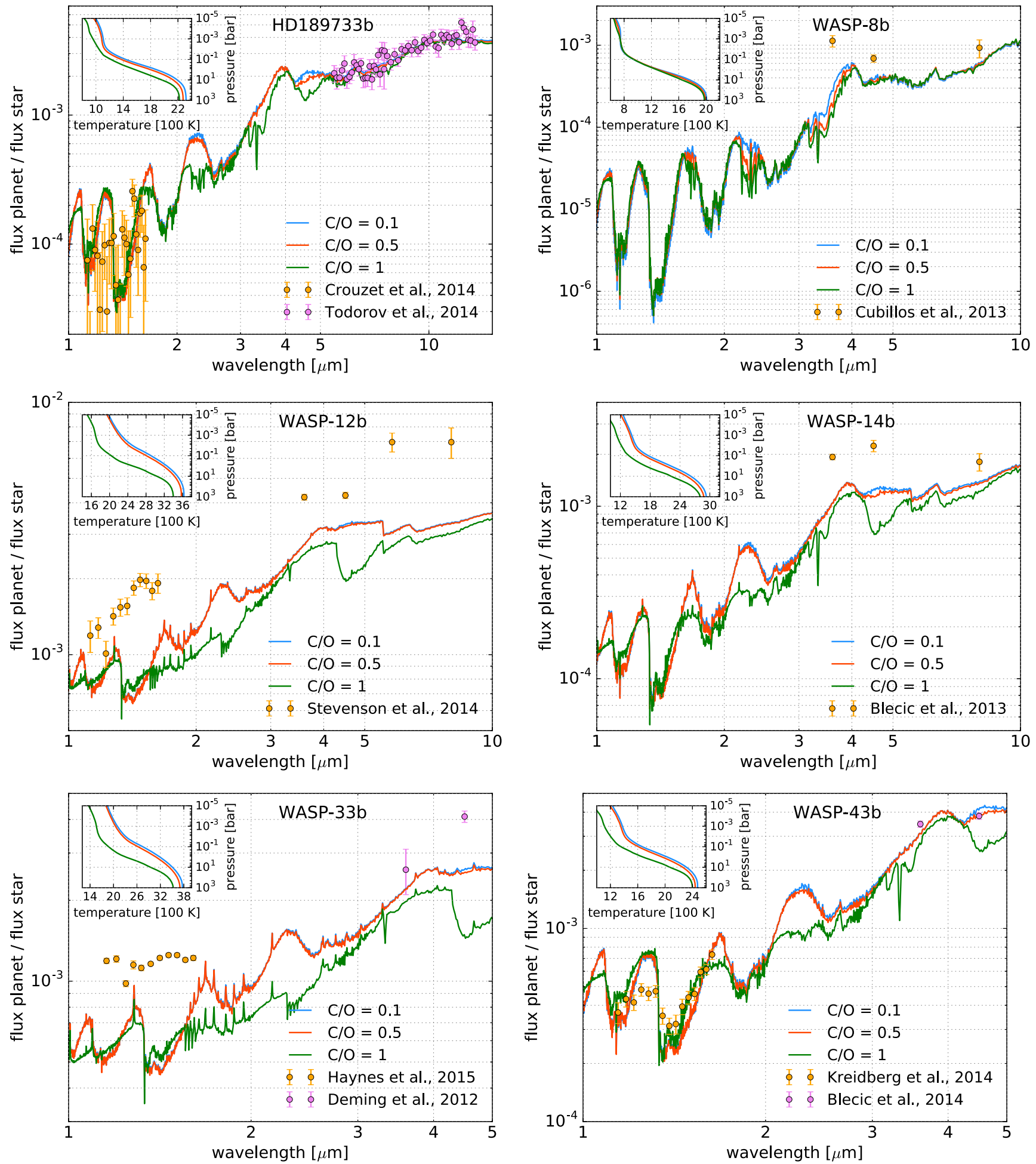

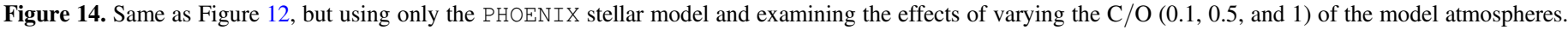

exoplanetary atmospheres. The work of Burrows et al. (2006, 2007, 2008) uses the accelerated lambda iteration method, originally developed for stellar atmospheres (Hubeny \& Lanz 1995). The work of Fortney et al. $(2005,2006,2008,2010)$ and Morley et al. $(2013,2015)$ uses an atmosphere modeling code and radiative transfer methods with a heritage from brown dwarf and solar system models (McKay et al. 1989; Toon et al. 1989; Burrows et al.
1997; Marley \& McKay 1999). Amundsen et al. (2014) recently implemented a radiative transfer code using the twostream approximation in the limit of pure absorption. Mollière et al. (2015) constructed a pure-absorption code using the "variable Eddington factor" method, which has a heritage from the study of stellar atmospheres (e.g., Auer \& Mihalas 1970) and protoplanetary disks (Dullemond 2002). Our approach and assembly of the various components (see 
above) and their collective implementation is a novel endeavor and we hope it will contribute to the advancement of this field.

\subsection{Discussion and Opportunities for Future Work}

In the current work, we have considered a small set of the four main infrared absorbers $\left(\mathrm{H}_{2} \mathrm{O}, \mathrm{CO}_{2}, \mathrm{CO}, \mathrm{CH}_{4}\right)$, and included the opacity associated with CIA from $\mathrm{H}_{2}-\mathrm{H}_{2}$ and $\mathrm{H}_{2}-$ He pairs. Future work should include more opacity sources, especially that associated with $\mathrm{C}_{2} \mathrm{H}_{2}$ and $\mathrm{HCN}$, if one is interested in $\mathrm{C} / \mathrm{O}>1$ models, and $\mathrm{Na}$ and $\mathrm{K}$ as these are major absorbers in the visible for very hot planets. Also important at the higher-temperature end of exoplanets is continuum absorption by electrons moving freely in the field or being decoupled from the shell of neutral atoms (e.g., $\mathrm{H}, \mathrm{He}$ ), molecules (e.g., $\mathrm{H}_{2}$ ), or ions (e.g., $\mathrm{H}^{-}$) (Sharp \& Burrows 2007). Furthermore, it is important to conduct a study examining the accuracy of the employed correlated- $k$ approximation for different combinations of molecular absorbers, since this could be a potential source of error-a study similar to Amundsen et al. (2016) for the random-overlap scheme. Another opportunity for future work is the inclusion of aerosols and clouds, whose proper implementation remains a subject of debate. Additionally, we will implement convective adjustment as the next step in sophistication and we plan to investigate the effect of disequilibrium chemistry (induced by both atmospheric motion and photochemistry) and radiative disequilibrium by coupling HELIOS to a chemical kinetics code and a general circulation model. Hot Jupiters are complex, threedimensional entities (e.g., Burrows et al. 2010) and interpreting them, on a detailed case-by-case basis, requires a threedimensional model (e.g., Kataria et al. 2015). The exact interpretation of the molecular abundances associated with the six hot Jupiters may be performed using an atmospheric retrieval code. HELIOS is a key component of the open-source Exoclimes Simulation Platform (exoclime.net), which includes a chemical kinetics code (Tsai et al. 2016), retrieval code (Lavie et al. 2016), and general circulation models (Mendonça et al. 2016; L. Grosheintz et al. 2017, in preparation). The upto-date version of HELIOS may be downloaded from its main repository github.com/exoclime/HELIOS and the version used to produce the results in this work is archived under the DOI: $10.5281 /$ zenodo.164176.

M.M., L.G., S.G., J.M., B.L., D.K., S.T., and K.H. thank the Swiss National Science Foundation (SNF), the Center for Space and Habitability (CSH), the PlanetS National Center of Competence in Research (NCCR), and the MERAC Foundation for partial financial support.

Software:HELIOS-K (Grimm \& Heng 2015; github.com/ exoclime/HELIOS-K), CUDA (Nickolls et al. 2008), PyCUDA (Klöckner et al. 2012), python (van Rossum 1995), scipy (Oliphant 2007), numpy (van der Walt et al. 2011), matplotlib (Hunter 2007).

\section{APPENDIX LICENSING AND PERMISSION TO USE THE TEA CODE}

We thank the developers of the Thermochemical Equilibrium Abundances (TEA) code (Blecic et al. 2015), initially developed at the University of Central Florida, Orlando,
Florida, USA. The Reproducible-Research Compendium (RRC) is available at github.com/exoclime/HELIOS. publications.

\section{REFERENCES}

Abramowitz, M., \& Stegun, I. A. 1972, Handbook of Mathematical Functions (New York: Dover)

Allard, F., \& Hauschildt, P. H. 1995, ApJ, 445, 433

Amundsen, D. S., Baraffe, I., Tremblin, P., et al. 2014, A\&A, 564, A59

Amundsen, D. S., Tremblin, P., Manners, J., Baraffe, I., \& Mayne, N. J. 2016, arXiv: 1610.01389

Anglada-Escudé, G., Rojas-Ayala, B., Boss, A. P., Weinberger, A. J., \& Lloyd, J. P. 2013, A\&A, 551, A48

Armstrong, B. H. 1969, JAtS, 26, 741

Auer, L. H., \& Mihalas, D. 1970, MNRAS, 149, 65

Barman, T. S., Hauschildt, P. H., \& Allard, F. 2005, ApJ, 632, 1132

Blecic, J., Harrington, J., \& Bowman, M. O. 2016, ApJS, 225, 4

Blecic, J., Harrington, J., Madhusudhan, N., et al. 2013, ApJ, 779, 5

Blecic, J., Harrington, J., Madhusudhan, N., et al. 2014, ApJ, 781, 116

Bouchy, F., Udry, S., Mayor, M., et al. 2005, A\&A, 444, L15

Boyajian, T., von Braun, K., Feiden, G. A., et al. 2015, MNRAS, 447, 846

Brown, T. M., Charbonneau, D., Gilliland, R. L., Noyes, R. W., \& Burrows, A 2001, ApJ, 552, 699

Burrows, A., Budaj, J., \& Hubeny, I. 2008, ApJ, 678, 1436

Burrows, A., Hubeny, I., Budaj, J., Knutson, H. A., \& Charbonneau, D. 2007 ApJL, 668, L171

Burrows, A., Marley, M., Hubbard, W. B., et al. 1997, ApJ, 491, 856

Burrows, A., Rauscher, E., Spiegel, D. S., \& Menou, K. 2010, ApJ, 719, 341

Burrows, A., Sudarsky, D., \& Hubeny, I. 2006, ApJ, 650, 1140

Cahoy, K. L., Marley, M. S., \& Fortney, J. J. 2010, ApJ, 724, 189

Chan, T., Ingemyr, M., Winn, J. N., et al. 2011, AJ, 141, 179

Chandrasekhar, S. 1960, Radiative Transfer (New York: Dover)

Charbonneau, D., Allen, L. E., Megeath, S. T., et al. 2005, ApJ, 626, 523

Charbonneau, D., Brown, T. M., Noyes, R. W., \& Gilliland, R. L. 2002, ApJ, 568,377

Collier Cameron, A., Guenther, E., Smalley, B., et al. 2010, MNRAS, 407, 507

Crouzet, N., McCullough, P. R., Deming, D., \& Madhusudhan, N. 2014, ApJ, 795,166

Cubillos, P., Harrington, J., Madhusudhan, N., et al. 2013, ApJ, 768, 42

de Kok, R. J., Brogi, M., Snellen, I. A. G., et al. 2013, A\&A, 554, A82

Deming, D., Fraine, J. D., Sada, P. V., et al. 2012, ApJ, 754, 106

Deming, D., Knutson, H., Kammer, J., et al. 2015, ApJ, 805, 132

Deming, D., Seager, S., Richardson, L. J., \& Harrington, J. 2005, Natur, 434,740

Dullemond, C. P. 2002, A\&A, 395, 853

Fortney, J. J., Cooper, C. S., Showman, A. P., Marley, M. S., \& Freedman, R. S. 2006, ApJ, 652, 746

Fortney, J. J., Lodders, K., Marley, M. S., \& Freedman, R. S. 2008, ApJ, 678,1419

Fortney, J. J., Marley, M. S., Lodders, K., Saumon, D., \& Freedman, R. 2005, ApJL, 627, L69

Fortney, J. J., Shabram, M., Showman, A. P., et al. 2010, ApJ, 709, 1396

Freedman, R. S., Marley, M. S., \& Lodders, K. 2008, ApJS, 174, 504

Gillon, M., Triaud, A. H. M. J., Fortney, J. J., et al. 2012, A\&A, 542, A4

Goody, R. M., \& Yung, Y. L. 1989, Atmospheric Radiation: Theoretical Basis (2nd ed.; New York: Oxford Univ. Press)

Grimm, S. L., \& Heng, K. 2015, ApJ, 808, 182

Hansen, C. J., Schwartz, J. C., \& Cowan, N. B. 2014, MNRAS, 444, 3632

Harpsøe, K. B. W., Hardis, S., Hinse, T. C., et al. 2013, A\&A, 549, A10

Hauschildt, P. H., Allard, F., \& Baron, E. 1999, ApJ, 512, 377

Haynes, K., Mandell, A. M., Madhusudhan, N., Deming, D., \& Knutson, H. 2015, ApJ, 806, 146

Hebb, L., Collier-Cameron, A., Loeillet, B., et al. 2009, ApJ, 693, 1920

Heng, K., \& Lyons, J. R. 2016, ApJ, 817, 149

Heng, K., Lyons, J. R., \& Tsai, S.-M. 2016, ApJ, 816, 96

Heng, K., Mendonça, J. M., \& Lee, J.-M. 2014, ApJS, 215, 4

Heng, K., \& Tsai, S.-M. 2016, ApJ, 829, 104

Hubeny, I., \& Lanz, T. 1995, ApJ, 439, 875

Hunter, J. D. 2007, CSE, 9, 90

Husser, T.-O., Wende-von Berg, S., Dreizler, S., et al. 2013, A\&A, 553, A6

Ingalls, J. G., Krick, J. E., Carey, S. J., et al. 2016, AJ, 152, 44

Joshi, Y. C., Pollacco, D., Collier Cameron, A., et al. 2009, MNRAS, 392, 1532

Kataria, T., Showman, A. P., Fortney, J. J., et al. 2015, ApJ, 801, 86 
Klöckner, A., Pinto, N., Lee, Y., et al. 2012, ParC, 38, 157

Kovács, G., Kovács, T., Hartman, J. D., et al. 2013, A\&A, 553, A44

Kreidberg, L., Bean, J. L., Désert, J.-M., et al. 2014, ApJL, 793, L27

Kurucz, R. L. 1979, ApJS, 40, 1

Lacis, A. A., \& Oinas, V. 1991, JGR, 96, 9027

Lavie, B., Mendonça, J. M., Mordasini, C., et al. 2016, arXiv:1610.03216

Lehmann, H., Guenther, E., Sebastian, D., et al. 2015, A\&A, 578, L4

Lodders, K., \& Fegley, B. 2002, Icar, 155, 393

Lodders, K., \& Fegley, B., Jr. 2006, in Chemistry of Low Mass Substellar Objects, ed. J. W. Mason (Chichester: Praxis), 1

Marley, M. S., \& McKay, C. P. 1999, Icar, 138, 268

Marley, M. S., \& Robinson, T. D. 2015, ARA\&A, 53, 279

McKay, C. P., Pollack, J. B., \& Courtin, R. 1989, Icar, 80, 23

Meador, W. E., \& Weaver, W. R. 1980, JAtS, 37, 630

Mendonça, J. M., Grimm, S. L., Grosheintz, L., \& Heng, K. 2016, ApJ, 829, 115

Mendonça, J. M., Read, P. L., Wilson, C. F., \& Lee, C. 2015, P\&SS, 105, 80

Mihalas, D. 1970, Stellar Atmospheres (San Francisco, CA: Freeman)

Miller-Ricci, E., \& Fortney, J. J. 2010, ApJL, 716, L74

Mollière, P., van Boekel, R., Dullemond, C., Henning, T., \& Mordasini, C. 2015, ApJ, 813, 47

Morley, C. V., Fortney, J. J., Kempton, E. M.-R., et al. 2013, ApJ, 775, 33

Morley, C. V., Fortney, J. J., Marley, M. S., et al. 2015, ApJ, 815, 110

Munari, U., Sordo, R., Castelli, F., \& Zwitter, T. 2005, A\&A, 442, 1127

Murphy, T., \& Meiksin, A. 2004, MNRAS, 351, 1430
Nickolls, J., Buck, I., Garland, M., \& Skadron, K. 2008, Queue, 6, 40

Oliphant, T. E. 2007, CSE, 9, 10

Pierrehumbert, R. T. 2010, Principles of Planetary Climate (Cambridge: Cambridge Univ. Press)

Queloz, D., Anderson, D. R., Collier Cameron, A., et al. 2010, A\&A, 517, L1

Richard, C., Gordon, I. E., Rothman, L. S., et al. 2012, JQSRT, 113, 1276

Rothman, L. S., Gordon, I. E., Babikov, Y., et al. 2013, JQSRT, 130, 4

Rothman, L. S., Gordon, I. E., Barber, R. J., et al. 2010, JQSRT, 111, 2139

Seager, S., \& Sasselov, D. D. 2000, ApJ, 537, 916

Sharp, C. M., \& Burrows, A. 2007, ApJS, 168, 140

Sneep, M., \& Ubachs, W. 2005, JQSRT, 92, 293

Southworth, J. 2010, MNRAS, 408, 1689

Spiegel, D. S., \& Burrows, A. 2010, ApJ, 722, 871

Stevenson, K. B., Bean, J. L., Madhusudhan, N., \& Harrington, J. 2014, ApJ, 791, 36

Stevenson, K. B., Harrington, J., Fortney, J. J., et al. 2012, ApJ, 754, 136

Sudarsky, D., Burrows, A., \& Hubeny, I. 2003, ApJ, 588, 1121

Todorov, K. O., Deming, D., Burrows, A., \& Grillmair, C. J. 2014, ApJ, 796, 100

Toon, O. B., McKay, C. P., Ackerman, T. P., \& Santhanam, K. 1989, JGR, 94, 16287

Tsai, S.-M., Lyons, J. R., Grosheintz, L., et al. 2016, arXiv:1607.00409 van der Walt, S. C. C., Colbert, S. C., \& Varoquaux, G. 2011, CSE, 13, 22 van Rossum, G. 1995, Python Tutorial, Report CS-R9526 